\title{
Analysis of far-UV data of central stars of planetary nebulae: Occurrence and variability of stellar winds ${ }^{\star}$
}

\author{
M. A. Guerrero ${ }^{1,2}$ and O. De Marco $^{2}$ \\ 1 Instituto de Astrofísica de Andalucía (IAA-CSIC), Glorieta de la Astronomía, 18008 Granada, Spain \\ e-mail: mar@iaa.es \\ 2 Department of Physics, Macquarie University, NSW 2109 Sydney, Australia \\ e-mail: orsola@science.mq.edu.au
}

Received 24 October 2012 / Accepted 6 March 2013

\begin{abstract}
The occurrence of stellar wind in the central star of a planetary nebula (CSPN) can be revealed by the presence of P Cygni profiles of high-excitation lines overimposed on its stellar continuum. We examined the entire Far-Ultraviolet Spectroscopic Explorer (FUSE) archive and merged all useful spectroscopic observations of CSPNe to produce the highest quality spectra that can be used to assess the frequency of stellar winds. Furthermore, the individual spectra of each CSPN were compared to search for variability in the P Cygni profile. P Cygni profiles of high-excitation lines have been found in 44 CSPNe, with a clear correlation between the ionization potential of the lines and the effective temperature of the star. We introduce a prescription to derive the terminal wind velocity $\left(v_{\infty}\right)$ from saturated and unsaturated P Cygni profiles and provide new values of $v_{\infty}$ for these stars. Another 23 CSPNe do not show P Cygni profiles, or else their data in the FUSE archive are not conclusive enough to determine the occurrence of P Cygni profiles. Variability in the P Cygni profile of high-excitation, far-UV lines is found for the first time in six CSPNe, namely Hen 2-131, NGC 40, NGC 1535, NGC 2392, Sp 3, and SwSt 1. This increases up to 13 the number of CSPNe with variable P Cygni profiles in the UV, including those previously reported using IUE or FUSE observations. Variability is seen primarily in the unsaturated P V and Si IV lines, but also in saturated C III and O VI lines. The CSPNe with variable P Cygni profiles have similar stellar properties (relatively $\operatorname{low} \log (g)$ and $\left.T_{\text {eff }}\right)$ that suggest they are less evolved CSPNe. Some of the CSPNe with variable P Cygni profiles show O vI lines, while their effective temperature is insufficient to produce this ion. We suggest that this ion is produced by Auger ionization from X-rays associated to shocks in their stellar winds, as is the case in massive OB stars of high ionization potential ions that cannot be abundantly produced by photoionizations.
\end{abstract}

Key words. line: profiles - stars: winds, outflows - planetary nebulae: general - ultraviolet: stars

\section{Introduction}

Planetary nebulae $(\mathrm{PNe})$ are a short stage in the stellar evolution of low- and intermediate-mass stars $\left(0.8-1.0 M_{\odot}<M_{\mathrm{i}}<\right.$ 8-10 $M_{\odot}$ ), when they have expelled most of their stellar envelopes at the end of the asymptotic giant branch (AGB) phase and start their evolution towards white dwarfs (WD). The formation and evolution of a PN is believed to be dominated by the fast stellar wind that emanates from the central star of the PN (CSPN) as it sweeps up the dense and slow AGB wind (Kwok et al. 1978) and evacuates a central cavity (see Balick \& Frank 2002, for a review). The interaction between this fast stellar wind and the slow AGB wind is so violent that the material in the stellar wind is shock-heated at high temperatures (Steffen et al. 2008), resulting in a hot bubble filled with X-ray emission (e.g., Kastner et al. 2000; Chu et al. 2001).

Most models of PN formation consider the stellar winds of CSPNe to be isotropic, homogeneous, and only variable on long time scales (e.g., Villaver et al. 2002; Perinotto et al. 2004). Since the radiatively-driven stellar winds of CSPNe and massive OB stars share many similarities (Prinja 1990), it can be

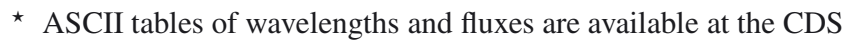
via anonymous ftp to cdsarc.u-strasbg. fr (130.79.128.5) or via http://cdsarc.u-strasbg.fr/viz-bin/qcat?]/A+A/553/A126 expected that the stellar winds of CSPNe also present the clumping and time variability unveiled in the wind of massive OB stars by changes in the profile of optical lines and by discrete absorption features (DACs) in the P Cygni profile of UV lines. The variability and clumping of the fast stellar wind can lead to insight into the interaction of the stellar wind with the nebular material, possibly requiring the reassessment of the wind-wind interaction in the models of PN formation and the revision of the mass-loss rate associated with these stellar winds. The hard X-ray emission observed in a few CSPNe, with NGC 6543 being the most prominent case (Guerrero et al. 2001), may be the smoking gun of shocks within the stellar winds of CSPNe as is seen in the strong stellar winds of OB stars (Lucy \& White 1980; Gayley \& Owocki 1995). An investigation of the frequency of wind variability and energies implied in the shocks in the limited sample of CSPNe with hard X-ray emission (Montez et al. 2010; Kastner et al. 2012; Guerrero et al., in prep.) will help the disambiguation between late-type companions and shock-in winds as the origin of the hard X-ray emission.

More important, the physical processes behind the wind variability, such as magnetic fields, stellar rotation, and binary companions, are assumed to play important roles in the shaping of PNe. Indeed, episodic, collimated mass-ejections, as are seen, e.g., in the central star of N66 in the LMC (Peña et al. 2008) or in the proto-PN M2-56 (Sánchez-Contreras et al. 2010), 
are assumed to shape the most axysimmetric PNe (Sahai \& Trauger 1998). The importance of stellar winds from CSPNe in the formation and evolution of PNe justifies detailed studies of their properties. For instance, wind variability may cause radial velocity $(\mathrm{RV}) \mathrm{RV}$ variations that interfere in the search for binary companions of CSPNe (De Marco et al. 2004). The detection of variability in the P Cygni profiles of a CSPN would make us question the interpretation that RV variations are due to the orbital motion. On the other hand, CSPNe that do not show variability in their P Cygni profiles will be cleared for future RV studies.

Earlier studies of the variability of P Cygni profiles of UV lines using International Ultraviolet Explorer (IUE) time series suggested that the stellar winds of a few CSPNe (BD+30 3639 , IC 4593, NGC 40, NGC 1535, NGC 2392, NGC 6543, and NGC 6826) can be variable (Patriarchi \& Perinotto 1995, 1997). The limited spectral resolution and sensitivity of IUE and the lack of complete time-series data prevented the search for patterns in the variability of the winds of these CSPNe. More recently, detailed studies of the time variability of the stellar winds of the CSPNe of IC 418, IC 2149, IC 4593, NGC 6543, NGC 6826 and Hen 2-138 have been obtained by Prinja et al. (2007, 2010, 2012b) using Far Ultraviolet Spectroscopic Explorer (FUSE) observations. These studies indicate that the stellar wind of these CSPNe are variable and, in the case of NGC 6543, this variability can be linked to the rotation of the star and so it has been used to measure its rotational velocity (Prinja et al. 2012a). Photometric and spectroscopic changes in the CSPNe of NGC 6826 have also been attributed to the stellar rotation (Handler et al. 2013).

Inspired by these works and with the aim of extending the results obtained in the 90's by Patriarchi \& Perinotto (1995, 1997), we present here a systematic study of all available FUSE observations of CSPNe to search for variability in the P Cygni profiles of far-UV lines of Galactic CPSNe. Since the available IUE and FUSE datasets are sparse in time, they are not suited to investigate in detail the time variability of these sources, but the detection of variability itself will aid in the selection of interesting targets for current and upcoming UV telescopes. In Sect. 2, we describe the available FUSE data and our sample of CSPNe. In Sect. 3, we present the data analysis and results, including an atlas of FUSE P Cygni profiles of CSPNe. In Sect. 4, we discuss individual CSPNe that show variable FUSE P Cygni profiles and describe further the observed variations. Finally, in Sect. 5 we present a discussion of the results, including the time scales and optical depths of the spectral variations observed in the FUSE $\mathrm{P}$ Cygni profiles of CSPNe, and how they correlate with hard $\mathrm{X}$-ray emission.

\section{UV observations}

\subsection{The FUSE observatory and FUSE archive}

Launched in June 1999, FUSE provides spectral coverage of the region 920-1190 A with a spectral resolving power $R \sim 20000$ (Moos et al. 2000; Sahnow et al. 2000). The FUSE instruments consist of four telescopes and Rowland circle spectrographs and two photon-counting detectors: two telescopes and diffraction gratings are made from silicon carbide ( $\mathrm{SiC}$ ), providing coverage of the short wavelength range, while the two other telescopes and gratings are lithium fluoride $(\mathrm{LiF})$ coated and cover the spectral region longward of $\sim 1020 \AA$. The combination of telescopes, gratings and detectors imply that a total of eight independent spectra are generated for each data set: two pairs of channels ( $\mathrm{SiC} 1$ and $\mathrm{SiC} 2$, and $\mathrm{LiF} 1$ and $\mathrm{LiF} 2)$, each of which is actually recorded by two detector segments (A and B). The channel and detector segment combinations correspond

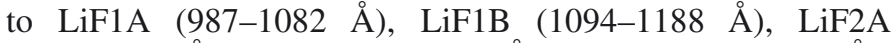
(1086-1187 ̊), LiF2B (979-1075 ̊), SiC1A (1004-1091 ), SiC1B (905-993 $\AA$ ), SiC2A (917-1006 A), and SiC2B (1016-1103 $\AA$ ). Therefore, flux at almost all wavelengths is recorded by at least two segments, with the short wavelength range (910-990 $\AA$ ) covered by the $\mathrm{SiC} 1 \mathrm{~B}$ and $\mathrm{SiC} 2 \mathrm{~A}$ spectra, the intermediate spectral range $(1015-1075 \AA)$ covered by the LiF1A, LiF2B, SiC1A, and SiC2B spectra, and the long wavelength range (1095-1185 $\AA$ ) covered by the LiF1B and LiF2A spectra.

Following the end of operations and decommissioning of FUSE in 2007, the entire FUSE science data were homogeneously reprocessed and archived at MAST, the Mikulski Archive for Space Telescopes, with the final version of the CalFUSE calibration pipeline software package, CalFUSE 3.2.3 (Dixon et al. 2007). This calibration software reprocessed FUSE data to remove instrumental effects, extract spectra, and apply wavelength and flux calibrations. The final reprocessing of the FUSE spectra permits the examination of a wealth of homogeneous, high-quality spectra covering the wavelength range from $920 \AA$ to $1190 \AA$.

\subsection{The sample}

We have searched the entire FUSE archive at MAST for observations of Galactic CSPNe showing P Cygni profiles of highexcitation UV lines. Following Guerrero et al. (2010), we have assembled a sample of 44 Galactic CSPNe with P Cygni profiles in FUSE observations. The names of these PNe and spectral types of their CSPNe are listed in Table 1, together with their stellar parameters (effective temperature, surface gravity, and mass loss rate) and the references from which these data have been compiled. An additional 23 Galactic CSPNe with FUSE data are found not to have P Cygni profiles, although the low continuum signal-to-noise ratio $(\mathrm{S} / \mathrm{N})$ prevents us from stating this with confidence for five of them (Hen 2-86, GJJC 1, M 4-18, NGC 3132, and NGC 3918).

We have then downloaded all available FUSE data of the objects with P Cygni profiles and screened them to select only the useful exposures. Exposures that showed an anomalously low count rate in the stellar continuum when compared to other exposures of the same object, were discarded. Likewise, LiF1B exposures affected by "the worm" in the 1140-1180 ̊ spectral range were disregarded for the analysis of the $\mathrm{P}$ Cygni profile of the C III $\lambda 1176$ multiplet. Table 2 summarizes the FUSE observations of CSPNe, including the observation ID, UT starting time of the first exposure, number of useful exposures, and total useful exposure time. The cadence among exposures is typically the total exposure time divided by the number of exposures, although it may change notably among different exposures.

As it could be expected, the final dataset is highly inhomogeneous. To better describe this dataset, we need to distinguish explicitly between an observation and an exposure. Typically, each observation consists of several exposures taken consecutively during a period of time. There are many CSPNe that have only one observation consisting of a number of useful exposures (e.g., NGC 2392), while there are some that have multiple observations taken at different epochs (e.g., NGC 6826). For the latter, the observing epochs are either close in time, taken within a few days, or separated in time by several months or even 
Table 1. CSPNe with FUSE observations.

\begin{tabular}{|c|c|c|c|c|c|c|c|c|c|c|c|}
\hline Name & Spectral type & $\begin{array}{c}T_{\text {eff }} \\
{[\mathrm{kK}]}\end{array}$ & $\begin{array}{c}\log (g) \\
{[\operatorname{cgs}]}\end{array}$ & $\begin{array}{c}\bar{M} \\
{\left[M_{\odot} \mathrm{yr}^{-1}\right]}\end{array}$ & Ref. & Name & Spectral type & $\begin{array}{c}T_{\text {eff }} \\
{[\mathrm{kK}]} \\
\end{array}$ & $\begin{array}{c}\log (g) \\
{[\mathrm{cgs}]} \\
\end{array}$ & $\begin{array}{c}\bar{M} \\
{\left[M_{\odot} \mathrm{yr}^{-1}\right]}\end{array}$ & Ref. \\
\hline \multicolumn{12}{|c|}{ CSPNe with P Cygni profiles } \\
\hline A 30 & [WC]-PG1159 & 115 & - & $5.0 \times 10^{-8}$ & 1 & NGC 246 & PG1159 & 150 & 5.7 & $1.3 \times 10^{-7}$ & 2,12 \\
\hline A 43 & Hybrid-PG1159 & 110 & 5.7 & $x=$ & 2 & NGC 1535 & $\mathrm{O}(\mathrm{H})$ & 75 & 5.0 & $1.4 \times 10^{-8}$ & 3 \\
\hline A 78 & [WC]-PG1159 & 113 & 5.7 & $4.7 \times 10^{-8}$ & 3 & NGC 2371-2 & [WC]-PG1159 & 135 & 6.3 & $7.8 \times 10^{-8}$ & 3 \\
\hline $\mathrm{BD}+30^{\circ} 3639$ & [WC9] & 47 & 4.2 & $1.6 \times 10^{-6}$ & 4 & NGC 2392 & $\mathrm{Of}(\mathrm{H})$ & 45 & 3.7 & $2.0 \times 10^{-8}$ & 10 \\
\hline Cn 3-1 & WELS & $\ldots$ & $\ldots$ & $\ldots$ & $\ldots$ & NGC 2867 & [WO1-2] & 141 & 6.0 & $5.8 \times 10^{-7}$ & 1 \\
\hline $\mathrm{Hb} 7$ & WELS & 50 & $\cdots$ & $2.9 \times 10^{-8}$ & 5 & NGC 5882 & $\mathrm{Of}(\mathrm{H})$ & 68 & 3.8 & . & $\ldots$ \\
\hline Hen 2-99 & [WC9] & 49 & $\ldots$ & $2.6 \times 10^{-6}$ & 6 & NGC 6058 & $\mathrm{O}(\mathrm{H})$ & 77 & 5.0 & $2.6 \times 10^{-9}$ & 10 \\
\hline Hen 2-131 & $\mathrm{Of}(\mathrm{H})$ & 33 & 3.1 & $3.5 \times 10^{-7}$ & 7 & NGC 6210 & $\mathrm{O}(\mathrm{H})$ & 75 & 5.0 & $2.4 \times 10^{-8}$ & 10 \\
\hline Hen $2-138$ & $\mathrm{Of}(\mathrm{H})$ & 29 & 3.0 & $1.2 \times 10^{-7}$ & 8 & NGC 6543 & Of-WR(H) & 60 & 4.7 & $1.1 \times 10^{-7}$ & 10 \\
\hline IC 418 & $\mathrm{Of}(\mathrm{H})$ & 39 & 3.7 & $7.2 \times 10^{-8}$ & 7 & NGC 6572 & WELS & 60 & 4.2 & $3.0 \times 10^{-8}$ & 9,13 \\
\hline IC 2149 & $\mathrm{Of}(\mathrm{H})$ & 42 & 3.6 & $2.0 \times 10^{-8}$ & 9 & NGC 6826 & $\mathrm{O} 3 \mathrm{f}(\mathrm{H})$ & 44 & 3.9 & $1.8 \times 10^{-7}$ & 7 \\
\hline IC 2448 & $\mathrm{O}(\mathrm{H})$ & 95 & 5.0 & $1.1 \times 10^{-8}$ & 10 & NGC 6891 & $\mathrm{O}(\mathrm{H})$ & 50 & 4.0 & $1.2 \times 10^{-7}$ & 14 \\
\hline IC 2501 & Emission line & $\ldots$ & $\ldots$ & $\ldots$ & 11 & NGC 7009 & $\mathrm{O}(\mathrm{H})$ & 87 & 4.9 & $2.9 \times 10^{-9}$ & 15,16 \\
\hline IC 2553 & Emission line & $\ldots$ & $\ldots$ & $\ldots$ & 11 & NGC 7094 & Hybrid PG1159 & 110 & 5.7 & $5.0 \times 10^{-8}$ & 12,17 \\
\hline IC 3568 & O5f & 50 & 4.0 & $3.0 \times 10^{-8}$ & 9 & NGC 7662 & $\ldots$ & 119 & 5.7 & $2.5 \times 10^{-9}$ & 10 \\
\hline IC 4593 & $\mathrm{O} 5 \mathrm{f}(\mathrm{H})$ & 41 & 4.0 & $2.3 \times 10^{-8}$ & 10 & PB 6 & [WO1] & 158 & $\ldots$ & $5.2 \times 10^{-7}$ & 1 \\
\hline IC 4776 & WELS & 65 & 5.1 & $1.4 \times 10^{-8}$ & 3 & PB 8 & {$[\mathrm{WN} / \mathrm{WC}]$} & 52 & $\ldots$ & $8.5 \times 10^{-8}$ & 18 \\
\hline IC 5217 & WELS & 95 & $\ldots$ & 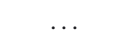 & $\ldots$ & RXJ2117.1+3412 & PG1159 & 170 & 6.0 & $1.0 \times 10^{-7}$ & 12 \\
\hline K $1-16$ & PG1159 & 140 & 6.4 & $2.5 \times 10^{-8}$ & 2,12 & Sp 3 & WELS & 50 & $\ldots$ & $7.1 \times 10^{-9}$ & 5 \\
\hline Lo 4 & PG1159 & 120 & 5.5 & $2.5 \times 10^{-8}$ & 1 & SwSt 1 & [WC9pec] & 40 & $\cdots$ & $1.9 \times 10^{-7}$ & 19 \\
\hline LSS 1362 & $\mathrm{O}(\mathrm{H})$ & 119 & 5.0 & $5.5 \times 10^{-9}$ & 10 & Tc 1 & $\mathrm{O}(\mathrm{H})$ & 35 & 3.6 & $2.1 \times 10^{-8}$ & 7 \\
\hline NGC 40 & [WC8] & 71 & 4.8 & $1.8 \times 10^{-6}$ & 4 & Vy $2-3$ & $\ldots$ & 34 & $\ldots$ & $\ldots$ & $\ldots$ \\
\hline \multicolumn{12}{|c|}{ CSPNe without P Cygni profiles } \\
\hline A 7 & $\mathrm{hgO}(\mathrm{H})$ & 99 & 7.0 & $\ldots$ & $\ldots$ & NGC 1360 & $\mathrm{O}(\mathrm{H})$ & 105 & 5.6 & $1.0 \times 10^{-10}$ & 10 \\
\hline A 31 & $\operatorname{hgO}(\mathrm{H})$ & 85 & 6.6 & $\ldots$ & $\ldots$ & NGC 3132 & $\mathrm{~A} 2 \mathrm{~V}$ & $\ldots$ & $\ldots$ & $\ldots$ & $\ldots$ \\
\hline A 35 & DAO & 80 & 7.7 & $\cdots$ & 20 & NGC 3211 & $\ldots$ & $\cdots$ & $\cdots$ & $\cdots$ & $\cdots$ \\
\hline A 39 & $\operatorname{hgO}(\mathrm{H})$ & 117 & 6.3 & $\ldots$ & $\ldots$ & NGC 3587 & $\operatorname{hgO}(\mathrm{H})$ & 94 & 6.9 & $\ldots$ & 24 \\
\hline DeHt 2 & $\mathrm{O}(\mathrm{H})$ & 117 & 5.6 & $\ldots$ & $\ldots$ & NGC 3918 & & 140 & 6.5 & $\ldots$ & $\ldots$ \\
\hline GJJC 1 & $\mathrm{sdO}$ & 75 & 5.0 & $\ldots$ & 21 & NGC 6720 & $\operatorname{hgO}(\mathrm{H})$ & 101 & 6.9 & $\ldots$ & $\ldots$ \\
\hline HDW 4 & $\operatorname{hgO}(\mathrm{H})$ & 47 & 7.9 & $\ldots$ & $\ldots$ & NGC 7293 & hgO(H) & 104 & 7.0 & $\ldots$ & 25 \\
\hline Hen 2-86 & [WC4] & 68 & $\ldots$ & $\cdots$ & 22 & Ps 1 & sdO & 39 & 3.9 & $\cdots$ & 26 \\
\hline Hen 3-1357 & .. & $\ldots$ & $\begin{array}{l}\cdots \\
\ldots\end{array}$ & $\begin{array}{l}\cdots \\
\ldots\end{array}$ & $\ldots$ & PuWe 1 & $\mathrm{hgO}(\mathrm{H})$ & 94 & 7.1 & $\ldots$ & $\ldots$ \\
\hline K $1-26$ & $\ddot{\mathrm{hgO}}(\mathrm{H})$ & $\cdots$ & $\ldots$ & $\cdots$ & $\cdots$ & Sh 2-174 & $\mathrm{hgO}(\mathrm{H})$ & 69 & 6.7 & $\ldots$ & $\ldots$ \\
\hline K 2-2 & hgO $(\mathrm{H})$ & 67 & 6.1 & $\cdots$ & & Sh 2-216 & $\mathrm{hgO}(\mathrm{H})$ & 83 & 6.7 & $\cdots$ & $\begin{array}{l}\cdots \\
\cdots\end{array}$ \\
\hline M4-18 & [WC10] & 31 & $\ldots$ & $\cdots$ & 23 & & & & & & \\
\hline
\end{tabular}

References. (1) Koesterke (2001); (2) Miksa et al. (2002); (3) Herald \& Bianchi (2004); (4) Marcolino et al. (2007a); (5) Gauba et al. (2001); (6) Leuenhagen et al. (1996); (7) Pauldrach et al. (2004); (8) Prinja et al. (2010); (9) Modigliani et al. (1993); (10) Herald \& Bianchi (2011); (11) Weidmann \& Gamen (2011); (12) Koesterke \& Werner (1998); (13) Méndez et al. (1990); (14) McCarthy et al. (1990); (15) Cerruti-Sola \& Perinotto (1989); (16) Iping et al. (2006); (17) Koesterke et al. (1998); (18) Todt et al. (2010); (19) De Marco et al. (2001); (20) Herald \& Bianchi (2002); (21) Harrington \& Paltoglou (1993); (22) Gesicki et al. (2006); (23) De Marco \& Crowther (1999); (24) Napiwotzki (1999); (25) Napiwotzki \& Schönberner (1995); (26) Rauch et al. (2002).

years. Obviously, these "sparse time series" are not always suited for the detailed study of time variability, but the availability of multiple exposures for one single observation at least allows us to search for rapid changes of the P Cygni profiles of the targeted UV lines.

\subsection{Data reduction}

Individual FUSE spectra of a same object typically display small shifts in wavelength caused by the apparent motion of a source in the instrument aperture. Such motion may even move the source partially outside the aperture, resulting in differences in the flux registered by each segment and channel. To correct for these effects, the individual FUSE spectra of an object have been shifted by forcing narrow $\mathrm{H}_{2}$, interstellar medium (ISM), and airglow (C I, O I, Ar I, Fe II, etc.) absorptions to agree in wavelength with those of the highest $\mathrm{S} / \mathrm{N}$ spectrum. Similarly, the flux of these spectra has been scaled so that the level of the stellar continuum agrees with that of the highest $\mathrm{S} / \mathrm{N}$ spectrum. Since the FUSE dispersion solution is highly non-linear and the vignetting of a source may affect the segments and channels defining different spectral ranges, this process has been applied locally to six spectral regions corresponding to the lines of (1) S VI $\lambda \lambda 933$, 944, (2) Ne VII $\lambda 973.3$ and C III $\lambda 977$, (3) O VI $\lambda \lambda 1032,1038$,
(4) S IV $\lambda 1073$, (5) Si III $\lambda \lambda 1108,1110,1113$, P V $\lambda \lambda 1118,1128$ and Si IV $\lambda \lambda 1122,1128$, and (6) C III $\lambda \lambda 1176$, implying that the shifts in wavelength and flux rescaling are fine tuned for each of these spectral ranges. We note that the shifts in wavelength between different spectra are typically small $\left(\lesssim 5 \mathrm{~km} \mathrm{~s}^{-1}\right.$, or $\lesssim 1.5$ pixels).

For most of the sources in our sample, the comparison among individual registered spectra resulted in differences well within the noise. This general agreement between spectra supports our procedure for shifting and scaling individual spectra. Exposures taken at similar epochs but with different channels that were found not to be significantly different were combined to increase the $\mathrm{S} / \mathrm{N}$. Finally, all spectra were merged together to build a "master" mean spectrum to which we compared individual spectra taken at different epochs.

\subsection{Near-UV data}

While near-UV IUE observations are available for a large sample of CSPNe, most of them have been already analyzed or compiled by Patriarchi \& Perinotto (1991). Their study can be complemented with more recent analyses of IUE data (Patriarchi \& Perinotto 1996; Feibelman 1997, 1998, 1999; Gauba et al. 2001; Marcolino et al. 2007b). When necessary, we adopted the 
Table 2. Summary of FUSE observations of CSPNe with P Cygni profiles.

\begin{tabular}{|c|c|c|c|c|c|c|c|c|c|}
\hline Name & Obs. ID & $\begin{array}{c}\text { Start time } \\
\text { date and UT }\end{array}$ & $\begin{array}{l}\text { Number of } \\
\text { exposures }\end{array}$ & $\begin{array}{c}\text { Net exposure } \\
\text { time } \\
{[\mathrm{ks}]}\end{array}$ & Name & Obs. ID & $\begin{array}{c}\text { Start time } \\
\text { date and UT }\end{array}$ & $\begin{array}{l}\text { Number of } \\
\text { exposures }\end{array}$ & $\begin{array}{c}\text { Net exposure } \\
\text { time } \\
{[\mathrm{ks}]}\end{array}$ \\
\hline A 30 & B0230101 & $2001-04-1000: 12: 51$ & 3 & 4.1 & Lo 4 & B0230201 & $2001-05-2609: 00: 03$ & 12 & 24.1 \\
\hline \multirow[t]{2}{*}{ A 43} & В0520201 & $2001-07-29$ 20:41:47 & 10 & 11.4 & LSS 1362 & C1770301 & 2002-03-08 15:53:37 & 15 & 7.9 \\
\hline & B0520202 & 2001-08-03 22:18:20 & 7 & 9.5 & & P3020701 & $2003-12-1121: 30: 43$ & 63 & 29.1 \\
\hline \multirow[t]{3}{*}{ A 78} & B1100101 & 2000-11-13 03:56:14 & 12 & 7.9 & NGC 40 & A0850101 & $2000-09-14$ 12:05:10 & 3 & 13.5 \\
\hline & B1100102 & 2001-07-02 22:09:52 & 19 & 9.4 & & A0850102 & $2000-12-1608: 31: 59$ & 14 & 26.6 \\
\hline & E1180101 & 2004-11-10 22:09:01 & 37 & 56.2 & NGC 246 & E1180201 & 2004-07-12 17:01:48 & 15 & 6.5 \\
\hline $\mathrm{BD}+30^{\circ} 3639$ & A0850301 & $2000-06-05$ 21:47:25 & 17 & 27.1 & NGC 1535 & P1150808 & 2001-10-05 17:17:25 & 2 & 6.6 \\
\hline Cn 3-1 & D0890401 & 2004-05-15 07:45:43 & 9 & 21.9 & & C1770101 & 2003-01-01 20:27:40 & 15 & 7.9 \\
\hline $\mathrm{Hb} 7$ & D0890201 & 2003-09-19 21:27:06 & 2 & 2.5 & NGC 2371-2 & P1330301 & $2000-02-2605: 04: 45$ & 4 & 5.3 \\
\hline Hen 2-99 & D0890101 & 2003-02-17 13:53:09 & 6 & 24.7 & NGC 2392 & B0320601 & $2001-02-21 \quad 16: 27: 25$ & 2 & 3.9 \\
\hline \multirow[t]{6}{*}{ Hen 2-131 } & P1930301 & 2000-06-28 21:06:44 & 5 & 6.1 & NGC 2867 & Z9110901 & $2003-02-22$ 12:07:50 & 4 & 14.9 \\
\hline & U1091902 & $2006-06-2806: 50: 16$ & 4 & 7.6 & & U1072501 & 2006-07-13 06:06:40 & 2 & 4.3 \\
\hline & U1091904 & 2006-06-29 22:53:21 & 3 & 9.9 & & U1072502 & 2007-06-30 12:31:47 & 5 & 12.6 \\
\hline & U1091905 & $2007-04-16$ 20:02:45 & 2 & 1.0 & NGC 5882 & B0690401 & 2002-08-14 15:01:12 & 2 & 3.3 \\
\hline & U1091906 & 2007-06-15 00:22:39 & 2 & 8.0 & & В0690402 & 2002-08-15 09:20:04 & 8 & 14.9 \\
\hline & U1091907 & 2007-06-16 04:14:03 & 9 & 11.9 & & B0690403 & 2002-06-16 13:41:18 & 5 & 10.0 \\
\hline \multirow[t]{12}{*}{ Hen $2-138^{a}$} & I8020201 & 1999-09-23 15:26:37 & 15 & 8.5 & NGC 6058 & В0320401 & $2001-03-26$ 22:20:56 & 2 & 4.4 \\
\hline & I8020202 & 1999-09-23 18:17:16 & 15 & 8.5 & NGC 6210 & A0850201 & 2000-08-02 05:38:26 & 4 & 6.6 \\
\hline & I8020203 & 1999-09-23 21:40:14 & 16 & 8.6 & NGC $6543^{a}$ & Q1080202 & 2001-10-01 21:52:01 & 9 & 3.8 \\
\hline & I9040401 & $1999-09-21 \quad 21: 15: 39$ & 2 & 8.2 & & F0340105 & 2007-01-13 03:01:24 & 26 & 11.8 \\
\hline & P1042801 & 2000-03-29 22:25:04 & 11 & 20.2 & & F0340106 & 2007-01-14 02:38:02 & 18 & 6.9 \\
\hline & S1010101 & 2000-07-13 09:06:18 & 8 & 2.1 & & F0340107 & 2007-01-15 02:14:23 & 19 & 8.2 \\
\hline & M1140901 & $2000-07-12$ 23:06:34 & 1 & 3.3 & & F0340108 & 2007-01-16 01:37:52 & 10 & 3.6 \\
\hline & S6013601 & 2002-04-21 06:55:48 & 2 & 7.7 & NGC 6572 & B0320201 & 2001-09-15 10:48:58 & 4 & 6.9 \\
\hline & M7276401 & $2007-02-1605: 05: 15$ & 2 & 1.8 & NGC 6826 & P1930401 & $2000-08-07$ 22:48:04 & 12 & 5.8 \\
\hline & M7276402 & 2007-02-17 03:05:27 & 2 & 1.7 & & D1200601 & 2003-06-23 19:56:26 & 2 & 4.3 \\
\hline & M7276403 & 2007-02-18 23:37:42 & 2 & 2.4 & & D1201601 & 2003-10-16 11:01:28 & 1 & 0.8 \\
\hline & M7276001 & 2007-04-19 00:30:46 & 2 & 2.7 & & F1600201 & 2006-11-06 23:12:27 & 5 & 2.1 \\
\hline IC 418 & P1151111 & $2001-12-02 \quad 02: 23: 33$ & 9 & 4.1 & & F1600202 & 2006-11-09 22:15:00 & 3 & 1.9 \\
\hline \multirow{3}{*}{ IC 2149} & P1041402 & $1999-11-25$ 17:31:13 & 7 & 16.2 & & F1600209 & 2007-01-09 17:33:48 & 12 & 5.3 \\
\hline & P1041401 & 1999-12-02 03:09:44 & 8 & 21.7 & NGC 6891 & В0320301 & 2001-09-15 02:26:52 & 6 & 7.4 \\
\hline & P1041403 & 2000-01-14 22:47:38 & 11 & 17.2 & NGC 7009 & I8011001 & 1999-11-02 01:02:13 & 2 & 1.9 \\
\hline \multirow[t]{5}{*}{ IC 2448} & B0320701 & 2002-03-07 00:32:47 & 3 & 11.5 & & X0140201 & 1999-11-02 07:36:07 & 12 & 5.8 \\
\hline & S7011102 & $2005-03-30$ 15:50:37 & 6 & 12.4 & & I8011002 & 1999-11-02 17:26:29 & 2 & 2.7 \\
\hline & S7011103 & 2005-03-31 05:42:49 & 5 & 18.1 & NGC 7094 & P1043701 & $2000-11-1308: 53: 28$ & 14 & 22.6 \\
\hline & U1072101 & 2006-01-20 01:34:40 & 7 & 23.9 & NGC 7662 & В0690301 & 2001-07-20 20:08:48 & 4 & 10.2 \\
\hline & U1072103 & $2007-06-3000: 10: 28$ & 5 & 14.8 & PB 6 & Z9111101 & 2003-02-20 15:13:54 & 7 & 19.5 \\
\hline IC 2501 & Z9110101 & $2003-02-23 \quad 06: 20: 22$ & 3 & 11.0 & PB 8 & Z9111301 & $2003-06-17$ 12:35:50 & 5 & 16.7 \\
\hline IC 2553 & Z9110301 & 2003-04-24 07:06:05 & 3 & 8.5 & RX J2117.1+3412 & P1320501 & 2000-07-16 03:44:57 & 18 & 8.2 \\
\hline \multirow[t]{4}{*}{ IC 3568} & P1042201 & 2000-03-02 00:49:26 & 7 & 20.0 & & D1800201 & 2003-06-21 13:50:31 & 13 & 6.4 \\
\hline & U1031401 & 2006-02-03 20:18:19 & 1 & 1.2 & & D1800202 & 2003-06-22 11:09:45 & 23 & 10.7 \\
\hline & U1031402 & 2006-02-04 23:58:51 & 1 & 1.2 & Sp 3 & В0320801 & 2001-08-18 01:49:57 & 7 & 12.0 \\
\hline & U1031404 & 2007-03-18 14:56:07 & 5 & 13.0 & SwSt 1 & B0690101 & 2001-08-21 01:17:09 & 11 & 23.1 \\
\hline \multirow[t]{2}{*}{ IC 4593} & B0320102 & 2001-08-03 15:28:55 & 4 & 1.9 & & B0690102 & 2001-08-22 10:34:39 & 7 & 15.3 \\
\hline & D1200301 & $2004-04-16$ 13:25:30 & 3 & 7.3 & Tc 1 & P1980304 & 2000-04-11 16:54:58 & 2 & 5.6 \\
\hline IC 4776 & P1330501 & $2000-05-2100: 02: 15$ & 2 & 6.2 & Vy $2-3$ & В0320501 & 2001-07-20 10:01:30 & 4 & 9.8 \\
\hline IC 5217 & Z9112401 & $2003-10-19$ 20:38:13 & 6 & 7.2 & & & & & \\
\hline \multirow[t]{5}{*}{ K $1-16$} & I8110310 & 1999-10-15 07:01:57 & 1 & 4.2 & & & & & \\
\hline & M1031001 & 2000-07-23 18:59:07 & 9 & 9.9 & & & & & \\
\hline & M1031002 & 2002-11-03 14:54:18 & 3 & 12.4 & & & & & \\
\hline & M1031005 & $2002-12-25$ 19:05:12 & 3 & 8.5 & & & & & \\
\hline & M7170101 & $2006-03-23$ 09:05:23 & 4 & 4.6 & & & & & \\
\hline
\end{tabular}

Notes. ${ }^{(a)}$ The FUSE spectra of Hen 2-138 and NGC 6543 have been thoroughly investigated by Prinja et al. (2007, 2010) and will not be discussed in the following sections.

information on the detection of P Cygni profiles and the values of $v_{\infty}$ provided by these authors after an examination of the original IUE data. We note that, in general, the spectral resolution and quality of the IUE data are far lower than those of FUSE data.

Near-UV HST STIS observations are only available for a small sample of CSPNe showing P Cygni profiles in FUSE data (see below), namely Hen 2-138, NGC 1535, and NGC 6543.

\section{Atlas of FUSE P-Cygni profiles of CSPNe}

Table 3 lists the CSPNe in our sample that show lines with P Cygni profiles in their FUSE spectra. In this table, the lines in the FUSE spectral range are marked by "..." if the P Cygni profile is absent, a " $\times$ " if a constant P Cygni profile is present, or with "var" if a variable P Cygni profile is observed. The normalized spectra of the P Cygni profiles of these CSPNe, obtained from the average of all useful spectra, are shown in Fig. 1. Since we are combining all available FUSE observations for every single object, we note that the resulting mean spectra presented here have the possible highest quality.

The FUSE spectral range contains P Cygni profiles of a variety of lines of different ions. The presence and shape of these line profiles carry information about the star and its wind properties, including the stellar effective temperature $\left(T_{\text {eff }}\right)$, wind ionization, mass-loss rate $(\dot{M})$, terminal velocity $\left(v_{\infty}\right)$, chemical composition, and even binarity. Variability of spectral lines that form in the stellar wind could be expected if the companion is in a close orbit, within the line formation region, and its orbital motion modifies the line profiles in a periodic way. This mechanism, which has been reported in the massive $\mathrm{O}$ star plus WR star binary $\gamma$ Vel (St.-Louis et al. 1993; De Marco 2002) could exist in binary CSPNe as well, particularly those with an extended atmosphere such as [WC] CSPNe. Only one [WC] CSPN has been discovered to be a close binary using periodic light variability ascribed to irradiation effects (PN G222.8-04.2, 
Table 3. FUSE lines and variable P Cygni profiles in CSPNe. ${ }^{a}$

\begin{tabular}{|c|c|c|c|c|c|c|c|c|c|c|}
\hline CSPN & $\begin{array}{c}\text { S VI } \\
\lambda \lambda 933,944 \AA\end{array}$ & $\begin{array}{l}\text { Ne VII } \\
\lambda 973 \AA\end{array}$ & $\begin{array}{c}\text { C III } \\
\lambda 977 \AA\end{array}$ & $\begin{array}{c}\text { O VI } \\
\lambda \lambda 1032,1038 \AA\end{array}$ & $\begin{array}{c}\text { S IV } \\
\lambda 1073 \AA\end{array}$ & $\begin{array}{c}\text { Si III } \\
\lambda \lambda 1108,1110,1113 \AA\end{array}$ & $\begin{array}{c}\mathrm{P} \mathrm{V} \\
\lambda 1118 \AA\end{array}$ & $\begin{array}{c}\text { Si IV } \\
\lambda 1122 \AA\end{array}$ & $\begin{array}{c}\text { C III } \\
\lambda 1175-1176 \AA\end{array}$ & Variability $^{b, c}$ \\
\hline A 30 & $\ldots$ & $x$ & $\ldots$ & $\times$ & $\ldots$ & $\ldots$ & $\ldots$ & $\ldots$ & $\ldots$ & $N: 1,2$ \\
\hline A 43 & $\ldots$ & $x$ & $\ldots$ & $\times$ & $\ldots$ & $\ldots$ & $\ldots$ & $\ldots$ & $\ldots$ & $N: 1,2$ \\
\hline A 78 & $\cdots$ & $\times$ & $\cdots$ & $\hat{x}$ & $\cdots$ & $\cdots$ & $\cdots$ & $\cdots$ & $\cdots$ & $N: 2$ \\
\hline $\mathrm{BD}+30^{\circ} 3639$ & $\ldots$ & $\ldots$ & $\ldots$ ? & $\ldots$ & $\times$ & $\ldots$ & $\times$ & $x$ & $\times$ & $N: 3$ \\
\hline $\mathrm{Cn} 3-1$ & $\ldots$ & $\ldots$ & $\ldots$ ? & $\times$ & $\times$ & $\ldots$ & $x$ & $\times$ & $\times$ & $N: 1$ \\
\hline $\mathrm{Hb} 7$ & $x$ & $\ldots$ & $\ldots$ & $x$ & $\ldots$ & $\ldots$ & $\ldots$ & $\ldots$ & $\ldots$ & $N: 1$ \\
\hline Hen 2-99 & $\ldots$ & $\ldots$ & $\ldots$ & $x$ & $x$ & $\ldots$ & $x$ & $x$ & $x$ & $N: 1$ \\
\hline Hen $2-131$ & $\ldots$ & $\ldots$ & $x$ & $x$ & var & var & var & var & var & $Y: \mathrm{R}, \mathrm{N}$ \\
\hline IC 418 & $\cdots$ & $\cdots$ & $x$ & var & var & $\ldots$ & var & var & var & $Y: \mathrm{R}, \mathrm{B}, \mathrm{E}$ \\
\hline IC 2149 & $\ldots$ & $\ldots$ & $x$ & var & $\ldots$ & $\ldots$ & var & $\ldots$ & var & $Y: \mathrm{R}, \mathrm{E}$ \\
\hline IC 2448 & $\ldots$ & $\cdots$ & $\ldots$ & $\times$ & $\cdots$ & $\ldots$ & $\ldots$ & $\ldots$ & $\ldots$ & $N: 2$ \\
\hline IC 2501 & $\times$ & $\cdots$ & $\ldots$ & $\times$ & $\cdots$ & $\ldots$ & $\times$ & $\ldots$ & $\ldots$ & $N: 1$ \\
\hline IC 2553 & $\ldots$ & $\ldots$ & $\ldots$ & $\times$ & $\ldots$ & $\ldots$ & $\ldots$ & $\ldots$ & $\ldots$ & $N: 1,2$ \\
\hline IC 3568 & $\times$ & $\ldots$ & $\ldots$ & $\times$ & $\cdots$ & $\cdots$ & $\cdots$ & $\cdots$ & $\cdots$ & $N: 3$ \\
\hline IC 4593 & $x$ & $\ldots$ & $x$ & var & $\times$ & $\ldots$ & var & $\ldots$ & var & $Y: \mathrm{R}, \mathrm{B}$ \\
\hline IC 4776 & $x$ & $\ldots$ & $\ldots$ & $\times$ & $\ldots$ & $\ldots$ & $\ldots$ & $\ldots$ & $\ldots$ & $N: 1$ \\
\hline IC 5217 & $\ldots$ & $\ldots$ & $\ldots$ & $x$ & $\ldots$ & $\ldots$ & $\ldots$ & $\ldots$ & $\ldots$ & $N: 1,2$ \\
\hline K 1-16 & $\ldots$ & $x$ & $\ldots$ & $x$ & $\ldots$ & $\ldots$ & $\ldots$ & $\ldots$ & $\ldots$ & $N: 3$ \\
\hline Lo 4 & $\ldots$ & $x$ & $\ldots$ & $x$ & $\ldots$ & $\ldots$ & $\ldots$ & $\ldots$ & $\ldots$ & $N: 1$ \\
\hline LSS 1362 & $\ldots$ & $\times$ & $\ldots$ & $x$ & $\ldots$ & $\ldots$ & $\ldots$ & $\ldots$ & $\ldots$ & $N: 3$ \\
\hline NGC 40 & $\ldots$ & $\ldots$ & $x$ & $\times$ & $\times$ & $\ldots$ & var & $\times$ & $x$ & $Y: \mathrm{R}, \mathrm{B}, \mathrm{E}$ \\
\hline NGC 246 & $\cdots$ & $\times$ & $\ldots$ & $x$ & $\ldots$ & $\cdots$ & $\ldots$ & $\ldots$ & $\ldots$ & $N: 3$ \\
\hline NGC 1535 & $\ldots$ ? & $\ldots$ & $\ldots$ & var & $\ldots$ & $\ldots$ & $\ldots$ & $\ldots$ & $\ldots$ & $Y: \mathrm{N}, \mathrm{B}$ \\
\hline NGC 2371-2 & $\ldots$ & $\times$ & $\ldots$ & $\times$ & $\ldots$ & $\ldots$ & $\ldots$ & $\ldots$ & $\ldots$ & $N: 1,2$ \\
\hline NGC 2392 & var & $\ldots$ & $x$ & var & $\cdots$ & $\cdots$ & var & $\times$ & $\cdots$ & $Y: \mathrm{R}, \mathrm{B}$ \\
\hline NGC 2867 & $\ldots$ & $\ldots$ ? & $\ldots$ & $\times$ & $\ldots$ & $\ldots$ & $\ldots$ & $\ldots$ & $\ldots$ & $N: 1,2$ \\
\hline NGC 5882 & $\times$ & $\ldots$ & $\ldots$ & $\times$ & $\ldots$ & $\ldots$ & $\ldots$ & $\ldots$ & $\ldots$ & $N: 1,2$ \\
\hline NGC 6058 & $\ldots$ & $\ldots$ & $\ldots$ & $\times$ & $\ldots$ & $\ldots$ & $\ldots$ & $\ldots$ & $\ldots$ & $N: 1$ \\
\hline NGC 6210 & $x$ & $\ldots$ & $\ldots$ & $x$ & $\ldots$ & $\ldots$ & $\ldots$ & $\ldots$ & $\ldots$ & $N: 3$ \\
\hline NGC 6572 & $\times$ & $\ldots$ & $\ldots$ & $\times$ & $\ldots$ & $\ldots$ & $\times$ & $\ldots$ & $\times$ & $N: 1$ \\
\hline NGC 6826 & var & $\ldots$ & var & var & $\ldots$ & $\ldots$ & var & $\ldots$ & var & $Y: \mathrm{R}, \mathrm{N}, \mathrm{B}$ \\
\hline NGC 6891 & $\times$ & $\ldots$ & $\ldots$ & $\times$ & $\ldots$ & $\ldots$ & $\times$ & $\ldots$ & $\ldots$ & $N: 1$ \\
\hline NGC 7009 & $\ldots$ & $\ldots$ & $\ldots$ & $\times$ & $\ldots$ & $\ldots$ & $\ldots$ & $\ldots$ & $\ldots$ & $N: 2$ \\
\hline NGC 7094 & $\ldots$ & $\times$ & $\ldots$ & $\times$ & $\ldots$ & $\ldots$ & $\cdots$ & $\ldots$ & $\ldots$ & $N: 1,2$ \\
\hline NGC 7662 & $\ldots$ & $\ldots$ & $\ldots$ & $x$ & $\cdots$ & $\cdots$ & $\cdots$ & $\cdots$ & $\cdots$ & $N: 3$ \\
\hline PB 6 & $\ldots$ & $\ldots$ ? & $\ldots$ & $\times$ & $\ldots$ & $\ldots$ & $\ldots$ & $\ldots$ & $\ldots$ & $N: 1,2$ \\
\hline PB 8 & $\times ?$ & $\ldots$ ? & $\ldots$ & $\times$ & $\times$ & $\ldots$ & $x$ & $x$ & $\times$ & $N: 1$ \\
\hline RX J2117 & $\ldots$ & $\times$ & $\ldots$ & $x$ & $\ldots$ & $\ldots$ & $\ldots$ & $\ldots$ & $\ldots$ & $N: 3$ \\
\hline Sp 3 & var & $\ldots$ & $\ldots$ & var & $\ldots$ & $\ldots$ & $\times$ & $\ldots$ & $\ldots$ & $Y: \mathrm{R}, \mathrm{B}$ \\
\hline SwSt 1 & $\ldots$ & $\ldots$ & $\ldots$ ? & $x$ & var & var & $x$ & var & var & $Y: \mathrm{R}, \mathrm{N}, \mathrm{B}$ \\
\hline Tc 1 & $\ldots$ & $\ldots$ & $\times$ & $x$ & $x$ & $\ldots$ & $\ldots$ & $\ldots$ & $\times$ & $N: 1$ \\
\hline Vy $2-3$ & $\times$ & $\ldots$ & $\ldots$ & $\times$ & $\ldots$ & $\ldots$ & $\ldots$ & $\ldots$ & $\ldots$ & $N: 1$ \\
\hline
\end{tabular}

Notes. ${ }^{(a)}$ The $\times$ sign indicates that the line shows a constant P Cygni profile, while "var" means that the P Cygni profile is variable. ${ }^{(b)}$ When no variability $(N)$ is detected, " 1 " means that the data may be insufficient or of low quality to search for variability, " 2 " that the P Cygni profile is saturated or absorbed and the search for variability may be insensitive, and " 3 " that the data have good quality, but no variability was detected. ${ }^{(c)}$ When variable P Cygni profiles $(Y)$, "R" means a ripple moving bluewards, "N" a narrow absorption, "E" variability of the emission region of the profile, and "B" variability at velocities bluer than $v_{\infty}$.

Hajduk et al. 2010), but it has not been monitored for wind variability. Wind variability of the $\gamma$ Vel type may affect this and other [WC] CSPNe, even if other binary-induced effects are not prominent.

An examination of Fig. 1 reveals that, for a single CSPN, the P Cygni profiles of different lines may be dramatically different. This is, for instance, the case of the C III $\lambda 977$, C III $\lambda 1176$, and S IV $\lambda 1073$ lines of Hen 2-131. The situation becomes more complicated when unsaturated line profiles, such as the O VI $\lambda \lambda 1031.9,1037.6, \mathrm{P} v \lambda 1118$, and Si IV $\lambda 1122$ lines of Hen 2-131, are considered. Since the distinct physical processes that dominate a line determine its shape, and therefore the observational indicators of $v_{\infty}$ (lines formed farther from the star tend to have higher $v_{\infty}$ ), it is worthwhile to describe in detail the properties and characteristics of the ions and lines observed by FUSE in CSPNe.

\subsection{Ions in the stellar wind of CSPNe with lines in the FUSE spectral range}

Table 4 provides the identifications and rest wavelengths for typical P Cygni line profiles present in the FUSE spectra of CSPNe.
This table includes also information on the electronic transition, the nature of the line, and its transition probability. The relative transition probability of multiplet lines provides an indication of their relative strength of the lines. We describe these lines in order of decreasing ionization potential (IP).

The highest ionization species with lines in the FUSE spectra of CSPNe is Ne VII (IP: $157.9 \mathrm{eV}$ ). The Ne VII $\lambda 973.3$ line has only recently been identified in the atmosphere of a number of extremely hot hydrogen-deficient WDs (Werner et al. 2004), and in the stellar wind of the hot CSPNe A 78, K 1-16, and NGC 2371-2 (Herald et al. 2005). We have also identified this feature in the FUSE spectra of A 30, A 43, Lo 4, LSS 1362, NGC 246, NGC 7094, and RX J2117.1+3412, thus increasing to 10 the number of CSPNe that show this line. Only the Ne VII P Cygni profiles of NGC 2371-2 and LSS 1362 are clearly saturated. This may also be the case for A 43 and Lo 4 although the large number of narrow absorptions over-imposed on the profile makes it hard to determine; the remaining objects show unsaturated P Cygni profiles. All the CSPNe with P Cygni profile in the Ne VII line have $T_{\text {eff }}>100000 \mathrm{~K}$ (Fig. 2), as such a high effective temperature is needed to produce a significant flux of photons with energies above the IP of $0.16 \mathrm{keV}$, i.e., in the $\mathrm{X}$-ray domain. The photospheric emission of K 1-16, NGC 246, 

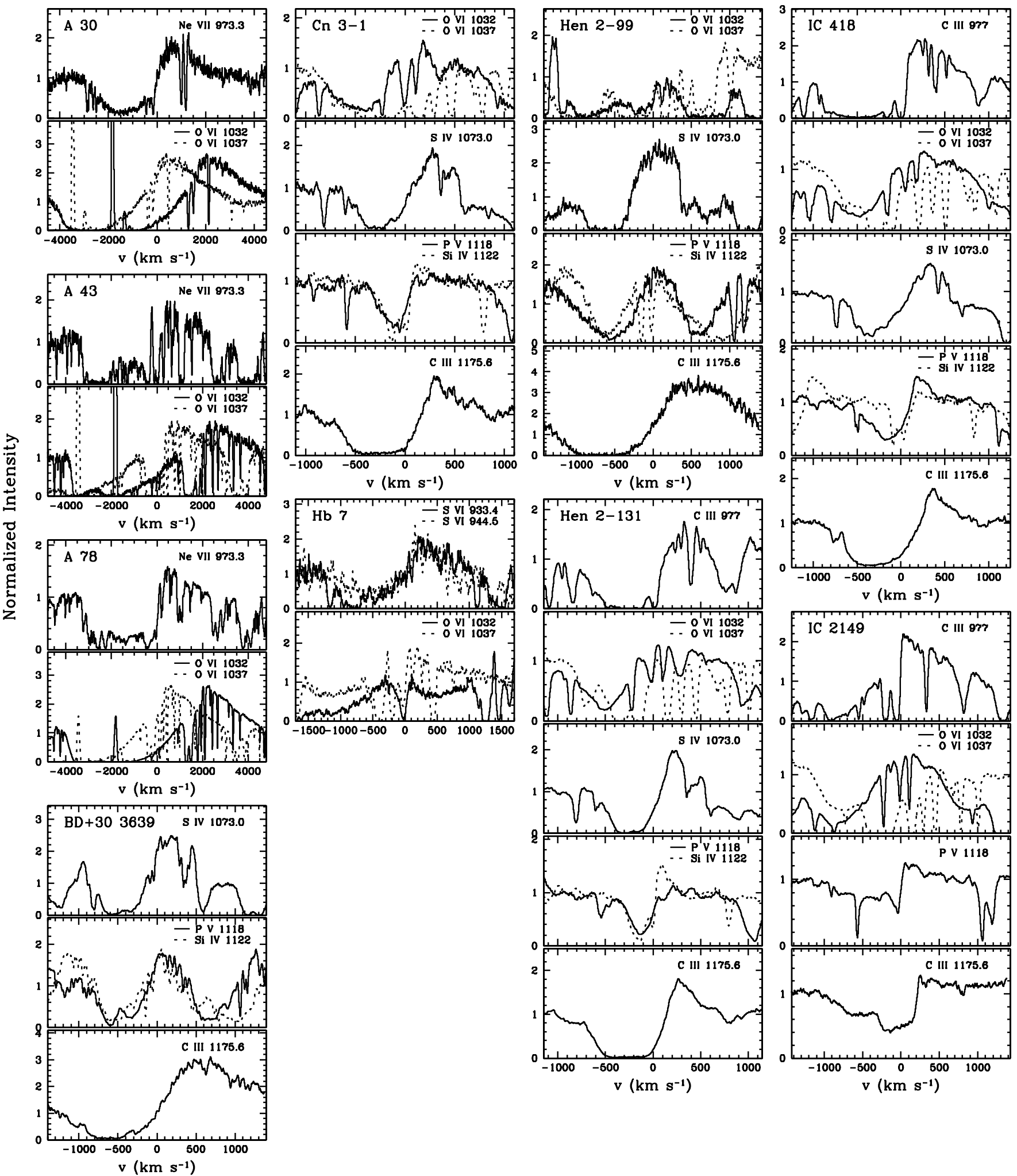

Fig. 1. Normalized P Cygni profiles in the FUSE spectral range.

NGC 2371-2, NGC 7094, and RX J2117.4+3412 is detected in soft X-rays (Motch et al. 1993; Hoare et al. 1995; Kastner et al. 2012), whereas A 30's central star's soft X-ray emission may have a different origin (Guerrero et al. 2012). We also note that all the stars that show Ne VII P Cygni profiles are CSPNe of the type PG 1159, with LSS 1362 being the only exception.
Otherwise, several stars hotter than $100000 \mathrm{~K}$ do not show clear evidence of the Ne VII line: A 7, A 39, DeHt 2, NGC 1360, NGC 2867, NGC 7293, NGC 7662, and PB 6. This may suggest that the occurrence of the Ne VII feature in the spectrum of a CSPN requires both high temperature (ionisation effect) and a hydrogen-deficient star (abundance effect). A high neon 

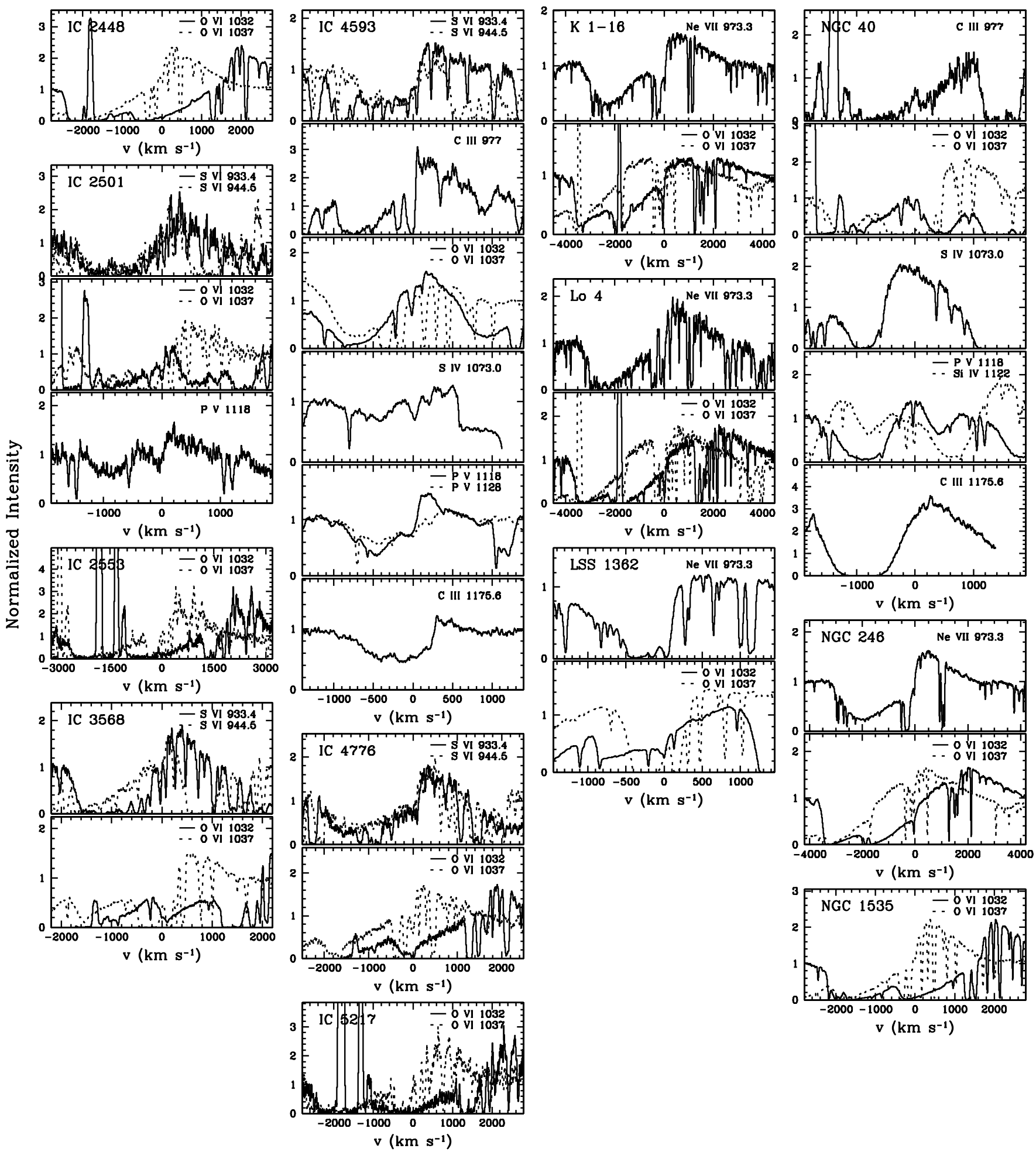

Fig. 1. continued.

abundance linked to hydrogen deficiency would provide support for the recent suggestion that [WC] stars and born-again CSPNe may be related with oxygen-neon novae (Lau et al. 2011).

The most common P Cygni profiles in the FUSE spectral range are those of the $O$ VI $\lambda \lambda 1031.9,1037.6$ (IP: $113.9 \mathrm{eV}$ ) resonance doublet, i.e., lines resulting from transitions to the ground level. While the presence of this doublet is widespread among our sample of CSPNe (Table 3), with BD+30 3639 being the only one that does not display it, the lines' characteristics still depend on the stellar effective temperature: not a single CSPN in our sample with $T_{\text {eff }}<50000 \mathrm{~K}$ shows saturated $\mathrm{O}$ VI P Cygni profiles, while it is saturated for most CSPNe with $T_{\text {eff }}>50000 \mathrm{~K}$. The chemical composition of the star may also play a role; [WR] CSPNe, with larger abundances of oxygen in their stellar winds, show a significant fraction of saturated O VI profiles. We note, however, that their effective temperatures are greater than $50000 \mathrm{~K}$ in most cases and so the possible dependence on oxygen abundance is hard 

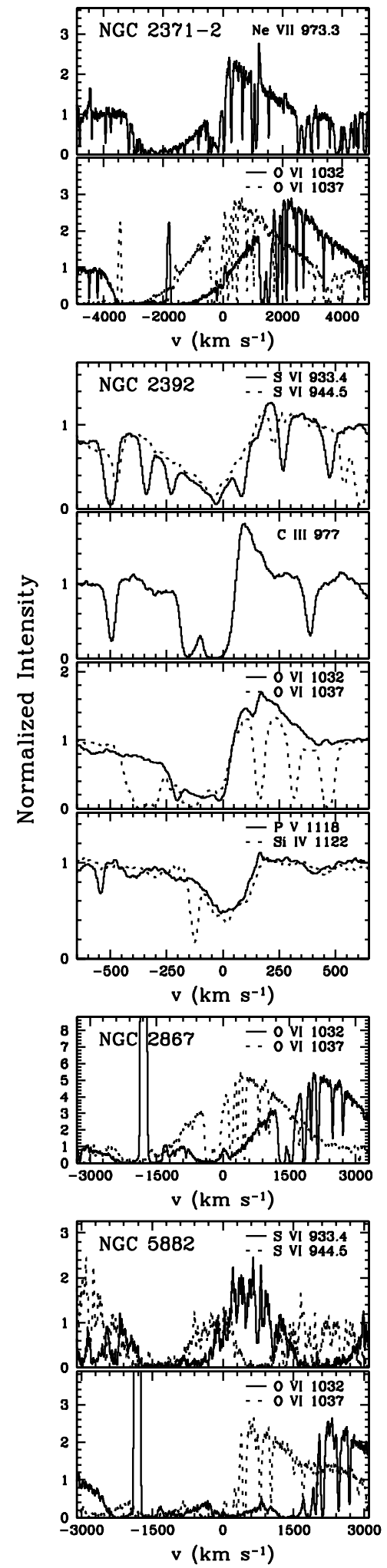
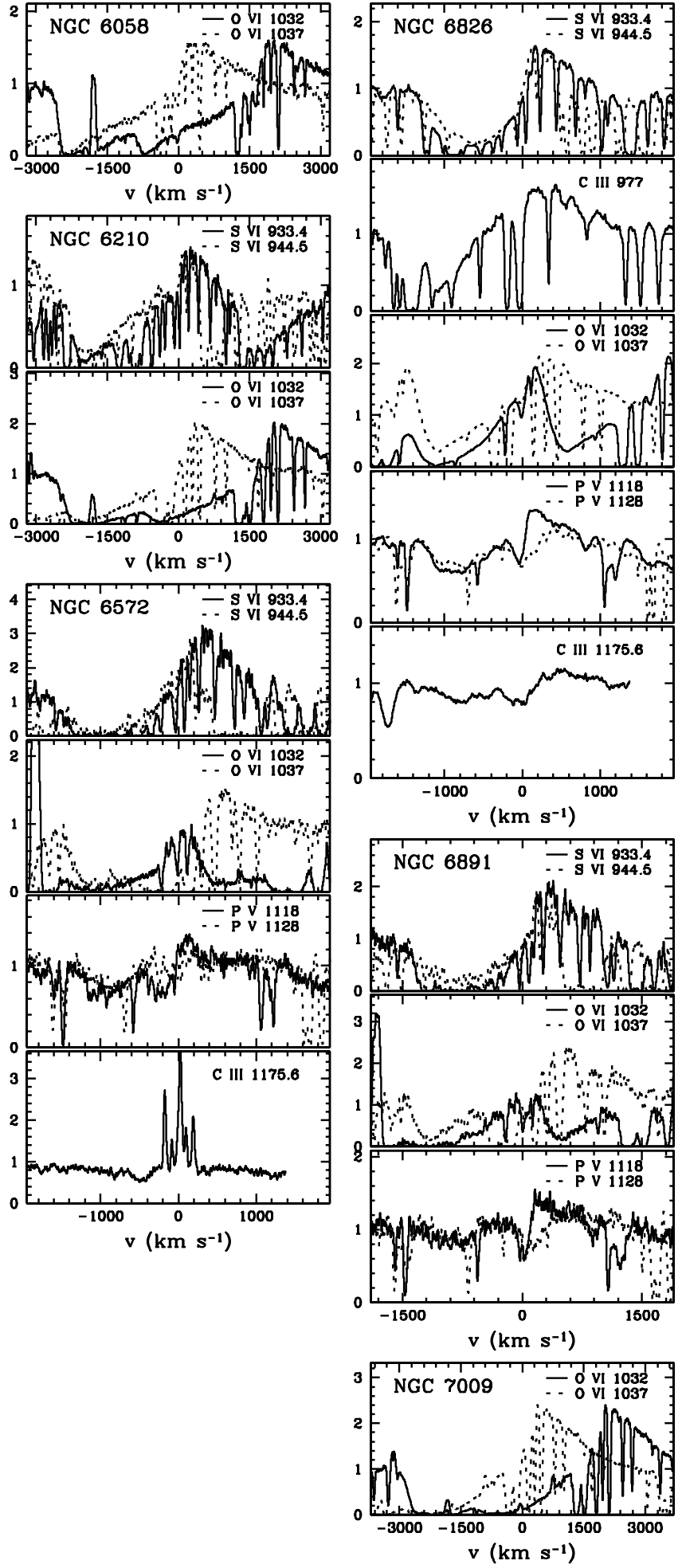
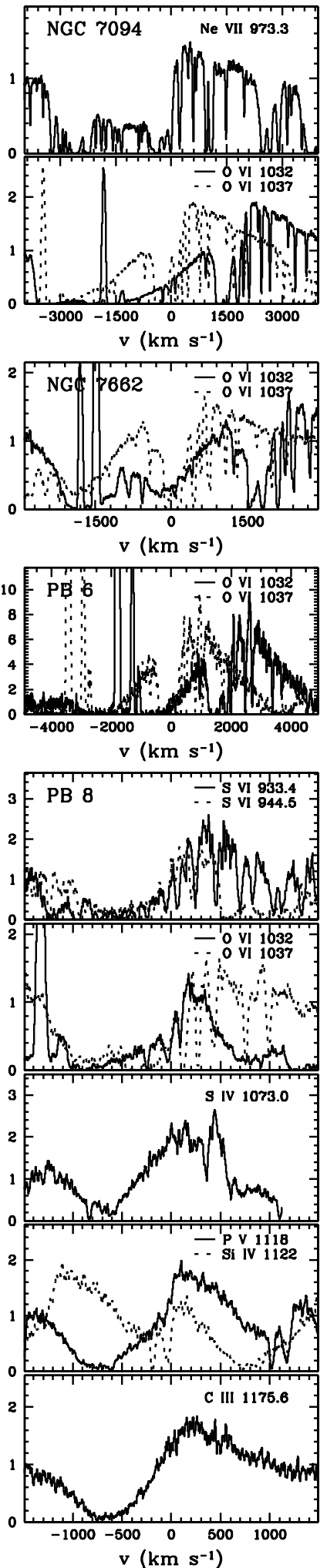

Fig. 1. continued.

to assess. Interestingly, the $\mathrm{O}$ VI P Cygni profiles of four hot CSPNe (K 1-16, LSS 1362, NGC 7662, and RX J2117.1+3412) are not saturated. These trends suggest that $\mathrm{O}$ VI is the dominant ionization stage for CSPNe with effective temperatures in the range $50000-100000 \mathrm{~K}$. The same statement cannot unambiguously be made for CSPNe with effective temperatures above
$100000 \mathrm{~K}$, because unsaturated O VI lines may result either from higher ionization stages being dominant (the IP of O VII is $138.12 \mathrm{eV}$ ) or from the reduced optical thickness of the wind, resulting from their low $\dot{M}$. Finally, we also remark that the measurement of terminal velocities greater than $\sim 1000 \mathrm{~km} \mathrm{~s}^{-1}$ from $\mathrm{O}$ VI lines is complicated by the separation of the doublet 

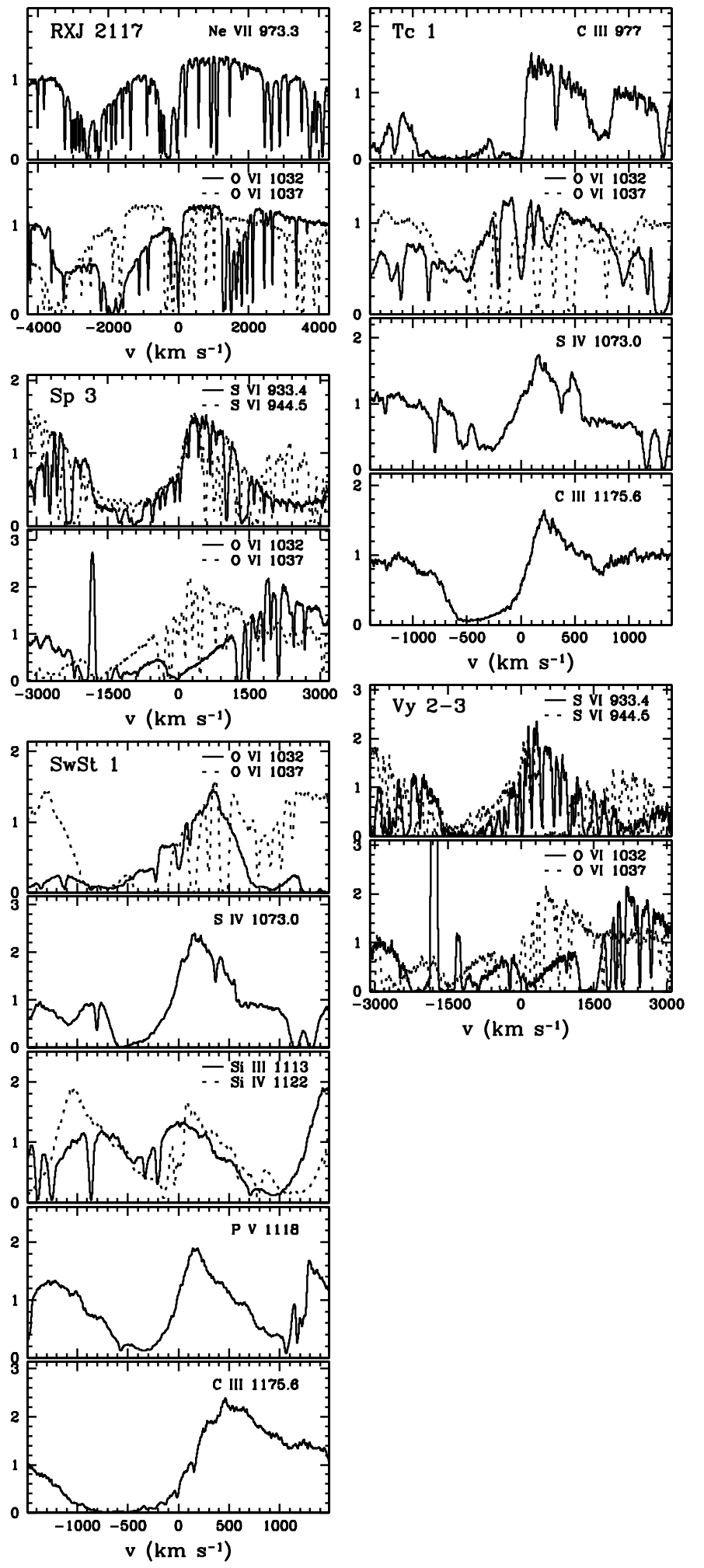

Fig. 1. continued.

components, $\sim 1650 \mathrm{~km} \mathrm{~s}^{-1}$, and by blending with the interstellar $\mathrm{Ly} \beta$ line, $\sim 1800 \mathrm{~km} \mathrm{~s}^{-1}$ bluewards of the $\mathrm{O}$ VI blue component at $1031.6 \AA$.

The S VI $\lambda \lambda 933.4,944.5$ (IP: $72.6 \mathrm{eV}$ ) resonance doublet is present in the short wavelength region of the FUSE spectra of 15 CSPNe. Since the IP of S VI is smaller than that of O VI, the $\mathrm{S}$ VI doublet is present in CSPNe with effective temperatures lower $\left(T_{\text {eff }}<87000 \mathrm{~K}\right)$ than those of the CSPNe with O VI lines (Fig. 2). We note that the spectral region of the S VI doublet is affected by a myriad of $\mathrm{H}$ I Lyman, He II Balmer, and $\mathrm{H}_{2}$ lines. Despite this difficulty, the P Cygni profiles of the two components can be used to lend weight to the determination of the line profile. Owing very likely to the sulfur abundance in the stellar wind being lower than that of oxygen, the $\mathrm{S}$ VI lines are saturated just in three CSPNe (IC 2501, NGC 5882, and NGC 6572) out of the 15 displaying it.

It is worth mentioning that Ne VII, O VI, and S VI have IPs well above that of He II (IP: $54.4 \mathrm{eV}$ ). The so-called "super ions" (Massa et al. 2003) with IPs above $54.4 \mathrm{eV}$ are not expected to be abundantly produced by photoionizations from the ground state in massive OB stars. The reason is the large bound-free absorption of $\mathrm{He}$ I of the ionizing stellar continuum flux shortward of $228 \AA$ (i.e., with energies greater than $54.4 \mathrm{eV}$ ). Thus, when "super-ions" are present in these stars, it is believed that they are produced by Auger ionizations of X-ray photons generated by shocks in the stellar wind (Cassinelli \& Olson 1979). Note, however, that CSPNe may have much higher effective temperatures than OB stars, and thus they can produce more ionizing photons with energies above the IP of He II. Indeed, the occurrence of the Ne VII or S VI lines is clearly limited to CSPNe with effective temperatures above or below $100000 \mathrm{~K}$, respectively. On the other hand, the $\mathrm{O}$ VI line is present over the entire range of effective temperatures. This issue will be discussed further in Sect. 6.3.

The P Cygni profile of the P V $\lambda \lambda 1118.01128 .0$ (IP: $51.4 \mathrm{eV}$ ) resonance doublet is typically unsaturated, given the relatively low phosphorus abundances. The red component of the doublet is usually blended with the Si IV $\lambda 1128.3$ line, and thus we have focused on the blue component. The P Cygni profiles of unsaturated $\mathrm{P} V \lambda 1118,1128$ lines have been singled out as a sensitive diagnostic of variable conditions in the wind of CSPNe (Prinja et al. 2007).

The S IV $\lambda \lambda 1073.0,1073.5$ lines (IP: $34.8 \mathrm{eV}$ ) are two finestructure lines. The strength of the blue component is $\sim 9$ times that of the red component (Table 4), and thus the blue component is expected to dominate the line profile. Although S IV and $S$ VI lines overlaps over a wide range of stellar temperatures (Fig. 2), there are only two CSPNe, IC 4593 and PB 8, that display P Cygni profiles of both species.

The Si IV $\lambda \lambda 1122.5,1128.3$ doublet (IP: $33.5 \mathrm{eV}$ ) is found among a significant number of CSPNe, all of them also presenting the $\mathrm{P}$ V doublet. As explained above, the red components of the Si IV and P V doublets are blended so these have not been used in our study. The Si IV $\lambda 1122$ is a line from a radiatively excited state. As the lower level of an excited state line is the upper level of a resonance line transition, its population depends strongly on the local radiation field and decreases rapidly with radial distance from the star (Olson 1981).

The C III $\lambda 1176$ (IP: $24.4 \mathrm{eV}$ ) is a triplet which has 5 separate, closely spaced levels, resulting in a multiplet composed of at least 6 bright lines. This line can act much like a resonance line in dense winds, scattering radiation every time $\mathrm{C}^{++}$is present. A prominent $\mathrm{P}$ Cygni feature is detected at the bluewards edge of this line in the [WR] central stars of BD+30 3639 , NGC 40 and PB 8, and the WELS of Sp 3. The origin of this line is uncertain, but it can be attributed to the C IV doublet $\lambda \lambda 1168.9,1169.0$, or to the N IV $\lambda \lambda 1168.6,1169.1,1169.5$ multiplet. The C III $\lambda 977$ resonance line is also present in some CSPNe, mostly (but not always) when the C III $\lambda 1176$ is detected.

Finally, the FUSE spectra of CSPNe also include the Si III $\lambda \lambda 1108.4,1110.0,1113.2$ triplet (IP: $16.3 \mathrm{eV})$. These lines 
Table 4. FUSE lines with P Cygni profiles in CSPNe.

\begin{tabular}{|c|c|c|c|c|c|}
\hline Ion & $\begin{array}{l}\lambda_{0} \\
{[\AA]}\end{array}$ & Transition & Term & $\begin{array}{c}\text { Transition probability } \\
{[\mathrm{s}]}\end{array}$ & Note \\
\hline C III & 977.03 & $1 s^{2} 2 s^{2}-1 s^{2} 2 s 2 p$ & ${ }^{1} \mathrm{~S}-{ }^{1} \mathrm{P}^{0}$ & $1.7 \times 10^{9}$ & Resonance line \\
\hline C III & 1174.933 & $1 \mathrm{~s}^{2} 2 \mathrm{~s} 2 \mathrm{p}-1 \mathrm{~s}^{2} 2 \mathrm{p}^{2}$ & ${ }^{3} \mathrm{P}^{1}-{ }^{3} \mathrm{P}^{2}$ & $3.3 \times 10^{8}$ & Multiplet \\
\hline $\mathrm{C}$ III & 1175.263 & $1 \mathrm{~s}^{2} 2 \mathrm{~s} 2 \mathrm{p}-1 \mathrm{~s}^{2} 2 \mathrm{p}^{2}$ & ${ }^{3} \mathrm{P}^{0}-{ }^{3} \mathrm{P}^{1}$ & $4.4 \times 10^{8}$ & Multiplet \\
\hline C III & 1175.590 & $1 \mathrm{~s}^{2} 2 \mathrm{~s} 2 \mathrm{p}-1 \mathrm{~s}^{2} 2 \mathrm{p}^{2}$ & ${ }^{3} \mathrm{P}^{1}-{ }^{3} \mathrm{P}^{1}$ & $3.3 \times 10^{8}$ & Multiplet \\
\hline C III & 1175.711 & $1 \mathrm{~s}^{2} 2 \mathrm{~s} 2 \mathrm{p}-1 \mathrm{~s}^{2} 2 \mathrm{p}^{2}$ & ${ }^{3} \mathrm{P}^{2}-{ }^{3} \mathrm{P}^{2}$ & $9.9 \times 10^{8}$ & Multiplet \\
\hline C III & 1175.987 & $1 \mathrm{~s}^{2} 2 \mathrm{~s} 2 \mathrm{p}-1 \mathrm{~s}^{2} 2 \mathrm{p}^{2}$ & ${ }^{3} \mathrm{P}^{1}-{ }^{3} \mathrm{P}^{0}$ & $1.3 \times 10^{9}$ & Multiplet \\
\hline C III & 1176.370 & $1 s^{2} 2 s 2 p-1 s^{2} 2 p^{2}$ & ${ }^{3} \mathrm{P}^{2}-{ }^{3} \mathrm{P}^{1}$ & $5.5 \times 10^{8}$ & Multiplet \\
\hline $\mathrm{Ne}$ VII & 973.302 & $1 \mathrm{~s}^{2} 2 \mathrm{~s} 2 \mathrm{p}-1 \mathrm{~s}^{2} 2 \mathrm{p}^{2}$ & ${ }^{1} \mathrm{P}^{0}-{ }^{1} \mathrm{D}$ & & Resonance line \\
\hline $\mathrm{O}$ VI & 1031.926 & $1 s^{2} 2 s-1 s^{2} 2 p$ & ${ }^{2} \mathrm{~S}-{ }^{2} \mathrm{P}^{0}$ & $4.2 \times 10^{8}$ & Resonance doublet \\
\hline O VI & 1037.617 & $1 s^{2} 2 s-1 s^{2} 2 p$ & ${ }^{2} \mathrm{~S}-{ }^{2} \mathrm{P}^{0}$ & $4.1 \times 10^{8}$ & Resonance doublet \\
\hline $\mathrm{PV}$ & 1117.977 & $2 p^{6} 3 s-2 p^{6} 3 p$ & ${ }^{2} \mathrm{~S}-{ }^{2} \mathrm{P}^{0}$ & $1.2 \times 10^{9}$ & Resonance doublet \\
\hline $\mathrm{PV}$ & 1128.008 & $2 p^{6} 3 s-2 p^{6} 3 p$ & ${ }^{2} \mathrm{~S}-{ }^{2} \mathrm{P}^{0}$ & $1.2 \times 10^{9}$ & Resonance doublet \\
\hline S IV & 1072.973 & $3 s^{2} 3 p-3 s 3 p^{2}$ & ${ }^{2} \mathrm{P}^{0}-^{2} \mathrm{D}$ & $2.0 \times 10^{9}$ & Fine-structure line \\
\hline S IV & 1073.518 & $3 s^{2} 3 p-3 s 3 p^{2}$ & ${ }^{2} \mathrm{P}^{0}-^{2} \mathrm{D}$ & $3.3 \times 10^{8}$ & Fine-structure line \\
\hline$S$ VI & 933.376 & $2 p^{6} 3 s-2 p^{6} 3 p$ & ${ }^{2} \mathrm{~S}-{ }^{2} \mathrm{P}^{0}$ & $1.7 \times 10^{9}$ & Resonance doublet \\
\hline S VI & 944.525 & $2 p^{6} 3 s-2 p^{6} 3 p$ & ${ }^{2} \mathrm{~S}-{ }^{2} \mathrm{P}^{0}$ & $1.6 \times 10^{9}$ & Resonance doublet \\
\hline Si III & 1108.358 & $3 s 3 p-3 s 3 d$ & ${ }^{3} \mathrm{P}^{0}-^{3} \mathrm{D}$ & $1.5 \times 10^{9}$ & Triplet \\
\hline Si III & 1109.970 & $3 s 3 p-3 s 3 d$ & ${ }^{3} \mathrm{P}^{0}-{ }^{3} \mathrm{D}$ & $2.1 \times 10^{9}$ & Triplet \\
\hline Si III & 1113.230 & $3 s 3 p-3 s 3 d$ & ${ }^{3} \mathrm{P}^{0}-{ }^{3} \mathrm{D}$ & $2.7 \times 10^{9}$ & Triplet \\
\hline Si IV & 1122.485 & $2 p^{6} 3 p-2 p^{6} 3 d$ & ${ }^{2} \mathrm{P}^{0}-^{2} \mathrm{D}$ & $2.1 \times 10^{9}$ & Doublet \\
\hline Si IV & 1128.340 & $2 p^{6} 3 p-2 p^{6} 3 d$ & ${ }^{2} \mathrm{P}^{0}-{ }^{2} \mathrm{D}$ & $2.5 \times 10^{9}$ & Doublet \\
\hline
\end{tabular}

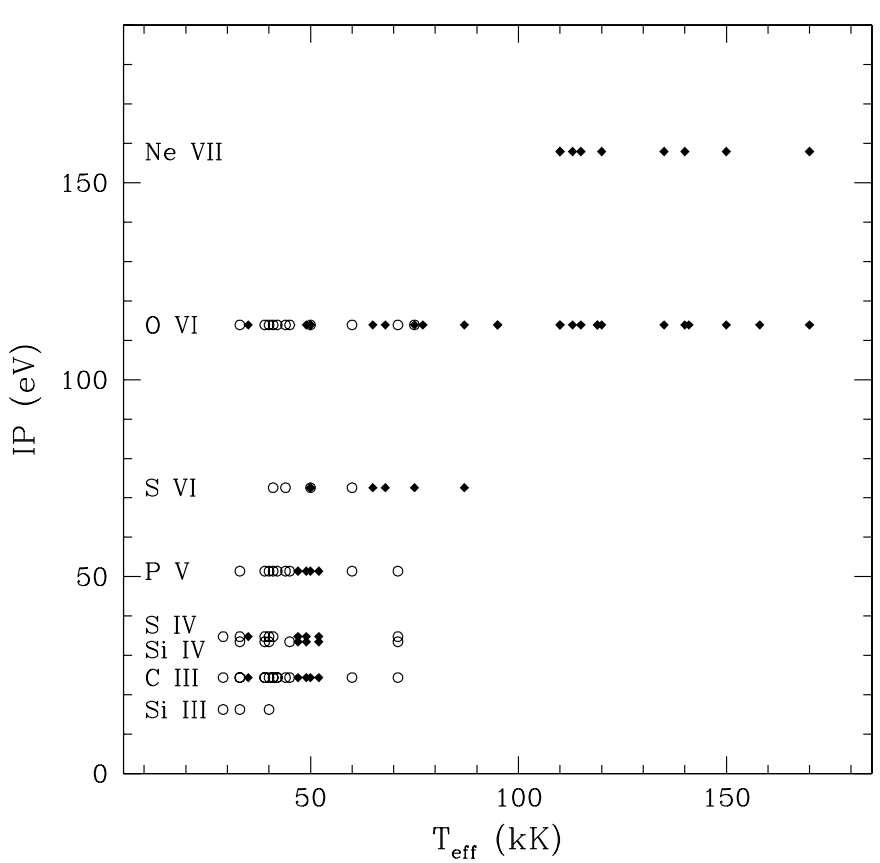

Fig. 2. Ionization potential of the lines with $\mathrm{P}$ Cygni profiles seen in the FUSE spectrum of a CSPN vs. its effective temperature. Objects that show line variability are shown as open circles and those that do not display variability as filled diamonds.

are only found in the CSPNe of Hen 2-131, Hen 21-138, and SwSt 1 , stars with $T_{\text {eff }} \leq 40000 \mathrm{~K}$, in line with the low IP of this ion. Interestingly, these CSPNe also show Si IV P Cygni profiles, indicating that both species are simultaneously present in their stellar winds.

\section{Terminal wind velocities}

The normalized spectra shown in Fig. 1 can be used to investigate the wind terminal velocities, $v_{\infty}$, of the CSPNe with FUSE
P Cygni profiles. Following the prescriptions by Prinja et al. (1990), we have determined the values of two observational indicators of $v_{\infty}$ : (1) the black velocity, $v_{\text {black}}$, the velocity of the blueward edge of the absorption region that has zero intensity; and (2) the edge velocity, $v_{\text {edge }}$, the velocity at which the profile rejoins the continuum level. In many cases, $v_{\text {black }}$ could not be determined because the line profile was not saturated. For these cases, we have defined a new observational indicator of $v_{\infty}$, the gray velocity, $v_{\text {gray }}$, as the velocity of the blueward edge of the absorption region that has the lowest intensity.

The values of these velocities are listed in Table 5. From the comparison between velocities derived from lines of a doublet (e.g., the O VI $\lambda 1031.9$ and $\lambda 1037.6$ lines, or the S VI $\lambda 933.4$ and $\lambda 944.5$ lines), the typical uncertainty in these velocities is estimated to be $10-40 \mathrm{~km} \mathrm{~s}^{-1}$, depending on the $\mathrm{S} / \mathrm{N}$, precise line shape, and blending with narrow telluric, $\mathrm{H}_{2}$, and atomic line absorptions. In certain cases, the blue edge of the line is heavily affected by these absorption lines, hampering the determination of the edge velocity. When this was the case, either an upper or a lower limit has been provided for the edge velocity.

An inspection of Table 5 reveals that the estimates of $v_{\text {edge }}$, $v_{\text {gray }}$, and $v_{\text {black }}$ implied from different lines can differ significantly from one line to another for a given CSPN, although these differences tend to be smaller for estimates of $v_{\text {black }}$. This is not completely unexpected as the atlas of FUSE P Cygni profiles of CSPNe in Fig. 1 reveals a variety of P Cygni profiles for the same CSPN. It is clear that these differences can result in uncertain estimates of the wind velocity.

As discussed by Abbott (1978) and Prinja et al. (1990), $v_{\text {black }}$ can be expected to be closer to $v_{\infty}$ than $v_{\text {edge. According to }}$ Table $5, v_{\text {edge }}$ is typically $150-200 \mathrm{~km} \mathrm{~s}^{-1}$ bluer than $v_{\text {black }}$, with some objects and lines for which the deviations are much greater. If we exclude objects with deviations greater than $1000 \mathrm{~km} \mathrm{~s}^{-1}$, the best linear to determine $v_{\text {black }}$ from $v_{\text {edge }}$ is:

$v_{\text {black }}=(0.939 \pm 0.017) \times v_{\text {edge }}-(175 \pm 32)\left[\mathrm{km} \mathrm{s}^{-1}\right]$,

which allows us to obtain an accurate estimate also for $v_{\infty}$. 
Table 5. Stellar wind velocities for CSPNe.

\begin{tabular}{|c|c|c|c|c|c|c|c|c|c|}
\hline Name & Line & $\begin{array}{c}v_{\text {edge }} \\
{\left[\mathrm{km} \mathrm{s}^{-1}\right]}\end{array}$ & $\begin{array}{c}v_{\text {gray }} \\
{\left[\mathrm{km} \mathrm{s}^{-1}\right]}\end{array}$ & $\begin{array}{c}v_{\text {black }} \\
{\left[\mathrm{km} \mathrm{s}^{-1}\right]}\end{array}$ & Name & Line & $\begin{array}{c}v_{\text {edge }} \\
{\left[\mathrm{km} \mathrm{s}^{-1}\right]}\end{array}$ & $\begin{array}{c}v_{\text {gray }} \\
{\left[\mathrm{km} \mathrm{s}^{-1}\right]}\end{array}$ & $\begin{array}{c}v_{\text {black }} \\
{\left[\mathrm{km} \mathrm{s}^{-1}\right]}\end{array}$ \\
\hline \multirow[t]{2}{*}{ A30 } & $\mathrm{Ne}$ VII & 2975 & 1950 & & NGC 40 & C III $\lambda 977$ & $\ldots$ & $\ldots$ & 910 \\
\hline & O VI & 4025 & ... & 2675 & & C III $\lambda 1176$ & 1400 & $\begin{array}{l}\cdots \\
\ldots\end{array}$ & 1150 \\
\hline \multirow[t]{2}{*}{ A 43} & $\mathrm{Ne}$ VII & $<3275$ & $\cdots$ & 3000 & & O VI & 1600 & $\ldots$ & 1280: \\
\hline & O VI & 3825 & $\ldots$ & 3600 & & $\mathrm{PV}$ & $>1400$ & 980 & \\
\hline \multirow[t]{2}{*}{ A 78} & $\mathrm{Ne}$ VII & $<3125$ & 3025 & & & S IV & 1330 & & 1000 \\
\hline & O VI & 4025 & $\ldots$ & 3450 & & Si IV & 970 & 800 & $\ldots$ \\
\hline \multirow[t]{4}{*}{$\mathrm{BD}+30^{\circ} 3639$} & C III $\lambda 1176$ & $\ldots$ & $\ldots$ & 710 & NGC 246 & $\mathrm{Ne}$ VII & 3170 & 2150 & $\ldots$ \\
\hline & $\mathrm{PV}$ & 800 & 420 & $\ldots$ & & O VI & 3580 & & 3390 \\
\hline & Si IV & 770 & 640 & & NGC 1535 & O VI & $>2080$ & 1825 & \\
\hline & S IV & $>740$ & $\ldots$ & 630 & NGC 2371-2 & $\mathrm{Ne}$ VII & 3030 & $\ldots$ & 2550 \\
\hline \multirow[t]{4}{*}{ Cn 3-1 } & C III $\lambda 1176$ & 690 & 370 & $\ldots$ & & $\mathrm{O}$ VI & 3990 & $\ldots$ & 3500 \\
\hline & $\mathrm{PV}$ & 370 & 180 & $\ldots$ & NGC 2392 & C III $\lambda 977$ & $>100$ & $\ldots$ & 65 \\
\hline & Si IV & 370 & $\ldots$ & & & O VI & $>270$ & $\ldots$ & 90 \\
\hline & S IV & $>480$ & & 380 & & P V & 140 & $\ldots$ & $\ldots$ \\
\hline \multirow[t]{2}{*}{$\mathrm{Hb} 7$} & O VI & $>1540$ & 1360 & $\ldots$ & & S VI & 330 & $\ldots$ & $\ldots$ \\
\hline & S VI & 1170 & 860 & & & Si IV & $<160$ & $\ldots$ & \\
\hline \multirow[t]{5}{*}{ Hen 2-99 } & C III $\lambda 1176$ & 1230 & $\ldots$ & 910 & NGC 2867 & O VI & 2560 & $\ldots$ & 2110 \\
\hline & O VI & $\ldots$ & $\ldots$ & 980: & NGC 5882 & O VI & $<2630$ & $\ldots$ & 1720 \\
\hline & $\mathrm{PV}$ & 1070 & 590 & $\ldots$ & & S VI & 2250 & $\ldots$ & 1870 \\
\hline & S IV & $>910$ & $\ldots$ & 830 & NGC 6058 & O VI & 2740 & $\ldots$ & 2600 \\
\hline & Si IV & 810 & 720 & $\ldots$ & NGC 6210 & O VI & 2530 & 2100 & $\ldots$ \\
\hline \multirow[t]{7}{*}{ Hen 2-131 } & C III $\lambda 977$ & $\ldots$ & $\ldots$ & 340 & & S VI & 2290 & $\ldots$ & 1910 \\
\hline & C III $\lambda 1176$ & $>680$ & $\cdots$ & 390 & NGC 6572 & C III $\lambda 1176$ & 600 & 320 & $\ldots$ \\
\hline & $\mathrm{O}$ VI & $\ldots$ & 560 & $\ldots$ & & O VI & 1420 & & 1190 \\
\hline & $\mathrm{PV}$ & 330 & 170 & $\ldots$ & & P V & 1210 & 1160 & $\ldots$ \\
\hline & S IV & $>480$ & $\ldots$ & 340 & & S VI & 1600 & & 1140 \\
\hline & Si III & $>290$ & 210 & $\ldots$ & NGC 6826 & C III $\lambda 977$ & 1200 & 760 & $\ldots$ \\
\hline & Si IV & 330 & 180 & $\ldots$ & & O VI & 1230 & $\ldots$ & 1100 \\
\hline \multirow[t]{6}{*}{ IC 418} & C III $\lambda 977$ & $\ldots$ & $\ldots$ & 510 & & P v & 1290 & 1080 & $\ldots$ \\
\hline & C III $\lambda 1176$ & 630 & & 390 & & S VI & $>1210$ & 710 & $\ldots$ \\
\hline & O VI & $>900$ & 530 & $\ldots$ & NGC 6891 & O VI & 1420 & 1230 & $\cdots$ \\
\hline & $\mathrm{PV}$ & $>310$ & 190 & $\ldots$ & & $\mathrm{PV}$ & 1260 & 910 & $\ldots$ \\
\hline & S IV & $>480$ & 420 & $\ldots$ & & S VI & $>990$ & $\ldots$ & 950 \\
\hline & Si IV & 120 & 100 & $\ldots$ & NGC 7009 & O VI & $>2870$ & $\ldots$ & 2540 \\
\hline \multirow[t]{4}{*}{ IC 2149} & C III $\lambda 977$ & $>1030$ & & 930 & NGC 7094 & $\mathrm{Ne}$ VII & 3330 & 1510 & \\
\hline & C III $\lambda 1176$ & 1030 & 180 & & & O VI & 3790 & & 3610 \\
\hline & O VI & 1190 & $\ldots$ & 890 & NGC 7662 & O VI & $<2580$ & 1640 & 列 \\
\hline & $\mathrm{PV}$ & 870 & $\ldots$ & 800 & PB 6 & O VI & 3140 & & 2980 \\
\hline IC 2448 & O VI & 2470 & $\ldots$ & 2280 & PB 8 & C III $\lambda 1176$ & & 1140 & 720 \\
\hline \multirow{3}{*}{ IC 2501} & O VI & 1400 & $\ldots$ & 1200 & & O VI & 1300 & $\ldots$ & 1000 \\
\hline & $\mathrm{PV}$ & 1320 & 1080 & & & $\mathrm{PV}$ & 1240 & $\ldots$ & 790 \\
\hline & S VI & 1430 & $\ldots$ & 1220 & & S IV & 1240 & 700 & $\ldots$ \\
\hline IC 2553 & O VI & 2700 & $\ldots$ & 2450 & & S VI & 1010 & 750 & $\ldots$ \\
\hline \multirow[t]{2}{*}{ IC 3586} & O VI & $>1700$ & 1640 & 200 & & Si IV & $\ldots$ & 470 & $\cdots$ \\
\hline & S VI & 1780 & $\ldots$ & 1580 & RXJ2117.1+3412 & $\mathrm{Ne}$ VII & 3490 & 3030 & $\ldots$ \\
\hline \multirow[t]{6}{*}{ IC 4593} & C III $\lambda 977$ & 960 & & 820 & & O VI & 3650 & 3140 & \\
\hline & C III $\lambda 1176$ & 720 & 420 & $\ldots$ & Sp 3 & O VI & $>1850$ & $\ldots$ & 1680 \\
\hline & O VI & 1150 & 860 & $\ldots$ & & S VI & 1940 & 1680 & $\ldots$ \\
\hline & $\mathrm{PV}$ & 920 & 480 & $\ldots$ & SwSt 1 & C III $\lambda 1176$ & 1500 & $\ldots$ & 770 \\
\hline & S IV & 1020 & 440 & $\ldots$ & & O VI & 1220 & 880 & $\ldots$ \\
\hline & S VI & 750 & 585 & $\ldots$ & & P V & $>760$ & 400 & $\ldots$ \\
\hline \multirow[t]{2}{*}{ IC 4776} & O VI & 2060 & & 1840 & & S IV & 770 & & 600 \\
\hline & S VI & 1960 & 1820 & $\ldots$ & & Si III & 610 & 450 & $\ldots$ \\
\hline IC 5217 & O VI & 2530 & $\ldots$ & 2360 & & Si IV & $>550$ & 330 & $\ldots$ \\
\hline K 1-16 & $\mathrm{Ne}$ VII & $>3000$ & 2410 & $\ldots$ & Tc 1 & C III $\lambda 977$ & 900 & $\ldots$ & 1050 \\
\hline & O VI & 3670 & 3550 & $\ldots$ & & C III $\lambda 1176$ & 790 & 540 & $\ldots$ \\
\hline Lo 4 & $\mathrm{Ne}$ VII & $>3100$ & 3000 & $\ldots$ & & O VI & 950 & 510 & $\ldots$ \\
\hline & O VI & 3830 & 3430 & $\ldots$ & & S IV & 440 & 390 & $\ldots$ \\
\hline LSS 1362 & $\mathrm{Ne}$ VII & $>520$ & $\ldots$ & 480 & Vy $2-3$ & O VI & 1740 & 1460 & $\ldots$ \\
\hline & O VI & $>500$ & $\ldots$ & $\ldots$ & & S VI & 1700 & $\ldots$ & 1460 \\
\hline
\end{tabular}

We note, however, that $v_{\text {black }}$ can only be equated to $v_{\infty}$ if the P Cygni profile is saturated. Unfortunately, this is not the case for many of the P Cygni profiles that have been examined. In such a case, we investigate the correlation between $v_{\text {gray }}$ and $v_{\text {edge. }}$. By definition, $v_{\text {gray }}$ is always redder than $v_{\text {edge }}$, as it is also the case for $v_{\text {black }}$. A linear fit also excluding objects with deviations greater than $1000 \mathrm{~km} \mathrm{~s}^{-1}$ provides the following relation between these two velocities:

$v_{\text {gray }}=(0.942 \pm 0.027) \times v_{\text {edge }}-(210 \pm 50)\left[\mathrm{km} \mathrm{s}^{-1}\right]$.

Such relationship is identical, within the uncertainties, to the relation between $v_{\text {black }}$ and $v_{\text {edge }}$, suggesting that $v_{\text {gray }}$ can be used as a proxy of $v_{\text {black }}$ in those cases when the line is not saturated.
In the following, we will adopt $v_{\text {black }}$ of saturated P Cygni profiles to be the terminal velocity of the wind. In such cases, the values of $v_{\text {gray }}$ derived from unsaturated P Cygni profiles, such as those of the Si IV $\lambda \lambda 1122.5,1128.3$, and P v $\lambda \lambda 1118.0,1128.0$, have not been used to determine $v_{\infty}$. Typically, the O VI $\lambda \lambda 1031.9,1037.6$ lines, when saturated, have been used to derive $v_{\text {black }}$ and these values have been assigned to $v_{\infty}$. In a number of cases, other saturated lines have been used instead: $\mathrm{S} \mathrm{IV}^{1}\left(\mathrm{BD}+30^{\circ} 3639\right.$, Cn 3-1, Hen 2-99, Hen 2-131, IC 2501, and NGC 40), C III $\lambda 977$ (IC 418, IC 2149, IC 4593, and NGC 2392), S VI $\lambda \lambda 933.4,944.5$ (IC 2501, IC 3568, NGC 6572, and Vy 2-3), and Ne VII 1973 (LSS 1362). The

1 There is, however, a caveat as the wavelength of this line has been assumed to be that of the dominant blue component. 
Table 6. CSPNe wind terminal velocities.

\begin{tabular}{|c|c|c|c|c|}
\hline Name & $\begin{array}{c}v_{\infty}^{\mathrm{FUSE}} \\
{\left[\mathrm{km} \mathrm{s}^{-1}\right]}\end{array}$ & $\begin{array}{c}v_{\infty}^{\mathrm{IUE}} \\
{\left[\mathrm{km} \mathrm{s}^{-1}\right]}\end{array}$ & $\begin{array}{c}v_{\infty}^{\text {model }} \\
{\left[\mathrm{km} \mathrm{s}^{-1}\right]}\end{array}$ & References \\
\hline A 30 & 3000 & 3400 & 4000 & 1 \\
\hline A 43 & 3000 & & $\ldots$ & $\ldots$ \\
\hline A 78 & 3450 & 3500 & 3200 & 2 \\
\hline $\mathrm{BD}+30^{\circ} 3639$ & 670 & 1000 & 700 & 3 \\
\hline Cn 3-1 & 380 & 330 & $\ldots$ & 3 \\
\hline $\mathrm{Hb} 7$ & 1360: & 1320 & $\ldots$ & 4 \\
\hline Hen 2-99 & 870 & $1000-2000$ & 900 & 5 \\
\hline Hen 2-131 & 370 & 850 & 450 & 6 \\
\hline Hen 2-138 & $\ldots$ & $\ldots$ & 300 & 7 \\
\hline IC 418 & 450 & 1050 & 700 & 8 \\
\hline IC 2149 & 930 & 1300 & 1000 & 8 \\
\hline IC 2448 & 2280 & & 2000 & 9 \\
\hline IC 2501 & 1210 & 1280 & $\ldots$ & $\ldots$ \\
\hline IC 2553 & 2450 & & $\ldots$ & $\ldots$ \\
\hline IC 3568 & 1580 & 1850 & 1730 & 10 \\
\hline IC 4593 & 820 & 1100 & 750 & 9 \\
\hline IC 4776 & 1840 & 1760 & 2300 & 2,6 \\
\hline IC 5217 & 2360 & & & $\ldots$ \\
\hline K 1-16 & 3550: & 3800 & 4000 & 11,12 \\
\hline Lo 4 & 3430: & $\ldots$ & 4000 & 1,13 \\
\hline LSS 1362 & 480 & $\ldots$ & 2400 & $\ldots$ \\
\hline NGC 40 & 1030 & 1600 & 1000 & 3 \\
\hline NGC 246 & 3390 & $>3300$ & 4000 & 11 \\
\hline NGC 1535 & 1825: & 2150 & 1950 & 2 \\
\hline NGC 2371-2 & 3500 & 4500 & 3700 & 2,14 \\
\hline NGC 2392 & 65 & 600 & 300 & 9 \\
\hline NGC 2867 & 2110 & $\ldots$ & 1800 & 1,15 \\
\hline NGC 5882 & 1720 & 1525 & . & $\ldots$ \\
\hline NGC 6058 & 2600 & & 2300 & 9 \\
\hline NGC 6210 & 2100 & 2350 & 2150 & 9 \\
\hline NGC 6572 & 1140 & 1550 & 1190 & 10 \\
\hline NGC 6543 & & & 1500 & 8 \\
\hline NGC 6826 & 1100 & 1600 & 1200 & 6 \\
\hline NGC 6891 & 950 & 1950 & & 16 \\
\hline NGC 7009 & 2540 & 2750 & 2770 & 17,18 \\
\hline NGC 7094 & 3610 & 3600 & 3500 & 11 \\
\hline NGC 7662 & 1640: & $\ldots$ & 2250 & 9 \\
\hline PB 6 & 2980 & $\ldots$ & 3000 & 1 \\
\hline PB 8 & 1000 & 1060 & 1000 & 19 \\
\hline RX J2117.1+3412 & 3140: & $\ldots$ & 3500 & 11 \\
\hline Sp 3 & 1680 & 1600 & $\ldots$ & 4 \\
\hline SwSt 1 & 880 & 1580 & 900 & 20 \\
\hline Tc 1 & 1050 & 1530 & 900 & 6 \\
\hline Vy $2-3$ & 1460 & $\ldots$ & $\ldots$ & $\ldots$ \\
\hline
\end{tabular}

Notes. The ":" sign indicates that these velocities have been derived from P Cygni profiles that are not completely saturated.

References. (1) Koesterke (2001); (2) Herald \& Bianchi (2004); (3) Marcolino et al. (2007a); (4) Gauba et al. (2001); (5) Leuenhagen et al. (1996); (6) Pauldrach et al. (2004); (7) Prinja et al. (2010); (8) Prinja et al. (2012b); (9) Herald \& Bianchi (2011); (10) Modigliani et al. (1993); (11) Koesterke \& Werner (1998); (12) Patriarchi \& Perinotto (1996); (13) Feibelman (1999); (14) Feibelman (1997); (15) Feibelman (1998); (16) McCarthy et al. (1990); (17) Cerruti-Sola \& Perinotto (1989); (18) Iping et al. (2006); (19) Todt et al. (2010); (20) De Marco et al. (2001).

C III $\lambda 1176$ multiplet is typically saturated, but $v_{\text {black }}$ is not easy to estimate because of the contribution to the profile of line components bluewards of the brightest component at $\lambda 1175.711 \AA$. To derive the value of $v_{\text {black }}$ for this line, we have computed the value of $v_{\text {black }}$ for each component of the multiplet and taken the weighted average using the transition probabilities as weights.

The FUSE spectra of Hb7, K 1-16, Lo 4, NGC 1535, NGC 7662, and RX J2117.1+3412 did not show any saturated $\mathrm{P}$ Cygni profiles. For those cases, we assumed $v_{\infty}$ to be the value of $v_{\text {gray }}$ of the most reliable line. The values of $v_{\infty}$ adopted from FUSE $v_{\text {black }}$ values are listed in Col. 2 of Table 6 together with those from IUE data (Col. 3) available in the literature as compiled by Patriarchi \& Perinotto (1991) or computed more recently by others (Patriarchi \& Perinotto 1996; Feibelman

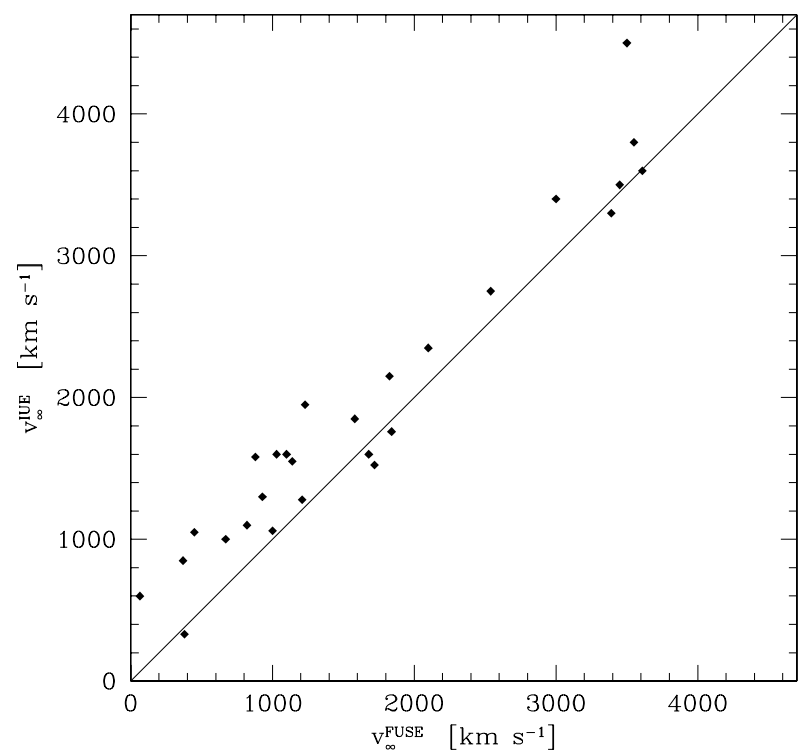

Fig. 3. Comparison of FUSE and IUE terminal velocities for the CSPNe listed in Table 6 . The solid line corresponds to the 1:1 correspondence.

1997, 1998, 1999; Gauba et al. 2001; Marcolino et al. 2007b) that assumed $v_{\infty}$ to be $v_{\text {edge. }}$. The terminal wind velocity derived from spectral fits is also listed in Col. 4, together with the references (Col. 5) from which those values have been compiled.

The comparison between FUSE and IUE terminal velocities (Table 6 and Fig. 3) implies that the latter are generally higher. The velocity difference is notably high, $\sim 500 \mathrm{~km} \mathrm{~s}^{-1}$, for objects with terminal velocities lower than $\sim 1000 \mathrm{~km} \mathrm{~s}^{-1}$ (Cn 3-1 being the only notable exception), suggesting that the spectral resolution of IUE is not sufficient to determine values of $v_{\infty}$ below $1000 \mathrm{~km} \mathrm{~s}^{-1}$. The typical difference between FUSE and IUE terminal velocities is similar to what is found between FUSE black and edge velocities, as can be expected because the IUE velocities compiled by Patriarchi \& Perinotto (1991) correspond to $v_{\text {edge }}$ rather than to $v_{\text {black }}$. This suggestion is further illustrated by Fig. 4 which compares our determination of $v_{\infty}$ for NGC $2392\left(65 \mathrm{~km} \mathrm{~s}^{-1}\right)$ and NGC $6826\left(1100 \mathrm{~km} \mathrm{~s}^{-1}\right)$ with those derived from IUE data (600 and $1600 \mathrm{~km} \mathrm{~s}^{-1}$, respectively). It is clear that the IUE velocities correspond to $v_{\text {edge }}$ and therefore must be discounted. This figure also sheds light on the large discrepancy between the IUE and FUSE terminal velocities of NGC 2392, as the terminal velocity in the former dataset has been associated to a shallow trough extending up to $\sim 600 \mathrm{~km} \mathrm{~s}^{-1}$ in the $\mathrm{N}$ V $\lambda \lambda 1238.8,1242.8$ and $\mathrm{C}$ IV $\lambda 1548.2$ lines (but not in the $\mathrm{C}$ IV red component at $\lambda 1150.8 \AA$ ).

\section{Wind variability}

\subsection{Detecting variability in FUSE P Cygni profiles of CSPNe}

The search for time variability in the P Cygni profiles of highexcitation UV lines in the FUSE spectra of a given CSPN has been performed by subtracting its "master" mean spectrum (Fig. 1) from individual exposures and visually inspecting the residuals to search for meaningful differences. This process has resulted in the identification of definite variations in the P Cygni profiles of the 10 CSPNe listed as variable in Table 3, of which 3, namely Hen 2-131, Sp 3 and SwSt 1, are found to be variable for the first time in this work. Since the line profile variations are not present in all of their P Cygni profiles, the lines that are found to be variable have been listed in this table as "var". 
M. A. Guerrero and O. De Marco: FUSE survey of CSPNe

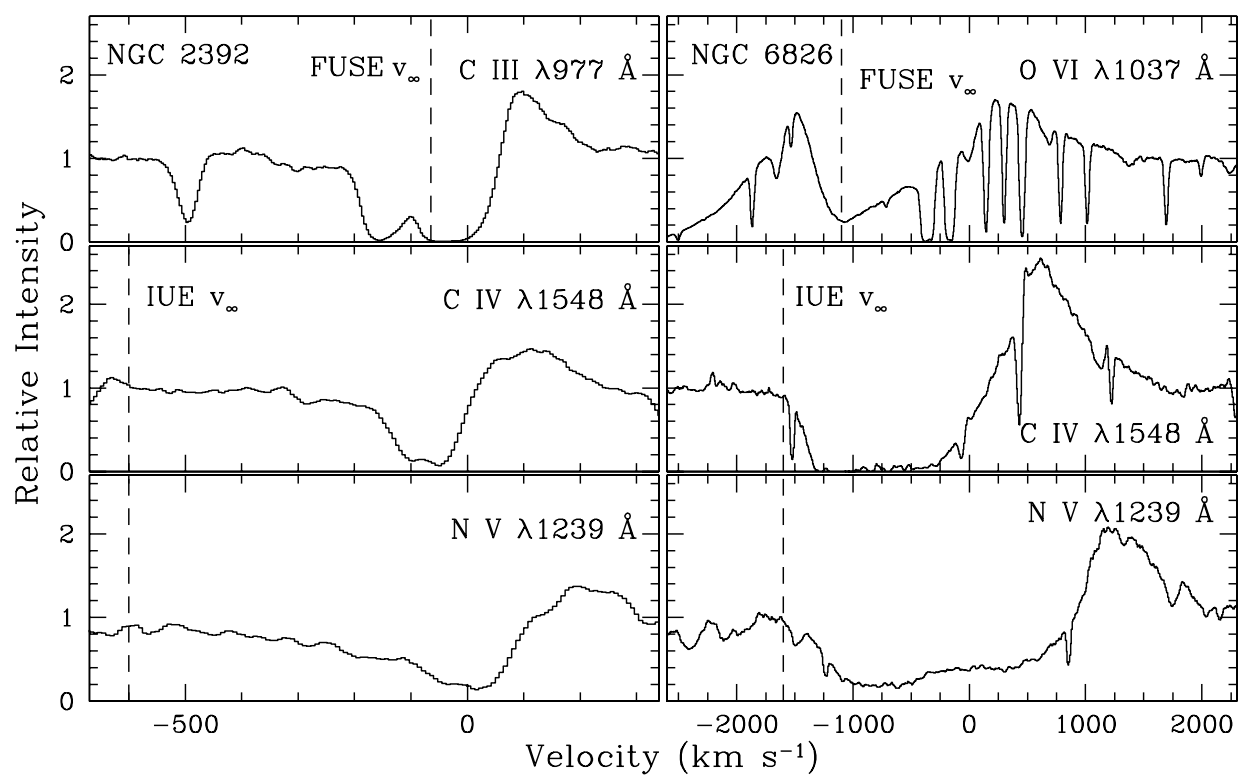

Fig. 4. Comparison of FUSE and IUE terminal velocities in the CSPNe of NGC 2392 (left) and NGC 6826 (right). The top panels show the normalized P Cygni profiles of the NGC 2392 C III $\lambda 977.0$ and NGC 6826 O VI $\lambda 1037.6$ lines derived from FUSE data. The middle and bottom panels show respectively the C IV $\lambda 1548.2$ and N v $\lambda 1238.8$ normalized P Cygni profiles of NGC 2392 (left) and NGC 6826 (right) derived from IUE data. The FUSE and IUE (Patriarchi \& Perinotto 1991) terminal velocities are shown by dashed vertical lines.

Table 3 also includes information on the CSPNe that do not show variability. These CSPNe can be classified into three different groups. The first group $(N=1$ in Table 3$)$ is composed of sources for which the number of individual spectra may be insufficient and/or the $\mathrm{S} / \mathrm{N}$ may be inadequate for the investigation of variability. The second group $(N=2$ in Table 3$)$ includes objects whose P Cygni profiles are strongly saturated or highly absorbed by multiple narrow lines, and thus they may be insensitive to line variations. It must be noted that the $\mathrm{P}$ Cygni profiles of some variable sources, while similarly affected by saturation and/or narrow line absorption, showed clear evidence of variability (e.g., NGC 1535). Finally, the third group $(N=3$ in Table 3) consists of sources that, having suitable P Cygni profiles for their study and a sufficient number of spectra with adequate $\mathrm{S} / \mathrm{N}$, lack variability in the particular FUSE observations presented in this paper. The latter are "bona-fide" candidates for non-variable winds, although due to the fragmented datasets presented here we cannot absolutely exclude that they are variable.

The variability detected in different lines for every CSPN in our study is illustrated in Fig. 5. The upper panels show the normalized averaged spectrum of selected individual P Cygni profiles in velocity space, while the lower panels display the temporal variance spectrum (TVS) that we define as the scaled unweighted variance spectrum:

$\operatorname{TVS}(\lambda)=\frac{1}{N} \frac{\sum\left[F_{i}(\lambda)-\bar{F}(\lambda)\right]^{2}}{\bar{F}(\lambda)}$,

where $F_{i}(\lambda)$ is the flux at wavelength $\lambda$ of the ith spectrum, $\bar{F}(\lambda)$ is the averaged spectrum at wavelength $\lambda$, and $N$ is the number of spectra (e.g., Howarth et al. 1993; Fullerton et al. 1996). As described by Howarth et al. (1993), the division by the averaged spectrum, $\bar{F}(\lambda)$, scales TVS so that it does not vary with wavelength in the absence of variability, regardless of the signal level. Actually, the plots in the lower panels of Fig. 5 have been divided by the continuum level at the spectral range of the line, $F_{\text {cont }}$, to provide a normalized quantity. These plots illustrate the different variability of these CSPNe and even the different variability of P Cygni profiles of different lines for the same CSPN. For instance, Hen 2-131 and IC 4593 show variability at different velocities in the $\mathrm{P} \mathrm{V}$ and $\mathrm{C}$ III lines. The variability can affect a significant fraction of the line profile (e.g., IC 2149) or be restricted to a narrow velocity range (e.g., NGC 1535).

It is interesting to compare the samples of FUSE (this paper) and IUE variable CSPNe (Patriarchi \& Perinotto 1995, 1997): IC 4593, NGC 40, NGC 1535, NGC 2392, NGC 6826, and NGC 6543 are both FUSE and IUE variable CSPNe, while NGC 246, NGC 6210, NGC 6572, and NGC 7009 are found to be non-variable. This agreement strengthens the statements about the variability of these CSPNe. Meanwhile, the limitations of the IUE and FUSE datasets used by these studies is proven by $\mathrm{BD}+30^{\circ} 3639$, which was found to be variable by IUE (Patriarchi \& Perinotto 1997), but non-variable by our FUSE study. On the other hand, the FUSE data reveal the variability of IC 418 and IC 2149 that was missed by IUE.

\subsection{Characteristics of the variable P Cygni profiles}

In order to investigate further the variability of the FUSE P Cygni profiles of CSPNe, we have plotted in Figs. 6-15 spectra derived from different individual exposures (bottom-panels) normalized by the mean spectra (top-panels). The P Cygni profiles shown in these figures exhibit different types of line variability. In Table 3 we label different types of variability: ripples (R), variability in the emission region $\left(\lambda>\lambda_{0}\right)$ of the profile $(E)$, and narrow features detected on the line profile trough $(\mathrm{N})$ or bluewards of $v_{\infty}(\mathrm{B})$. The most common variable features are broad ripples $(\mathrm{R})$ moving bluewards that are in many aspects similar to the DACs reported in the P Cygni UV profiles of massive OB stars. The velocities and time scales of individual features are discussed below for each object. 

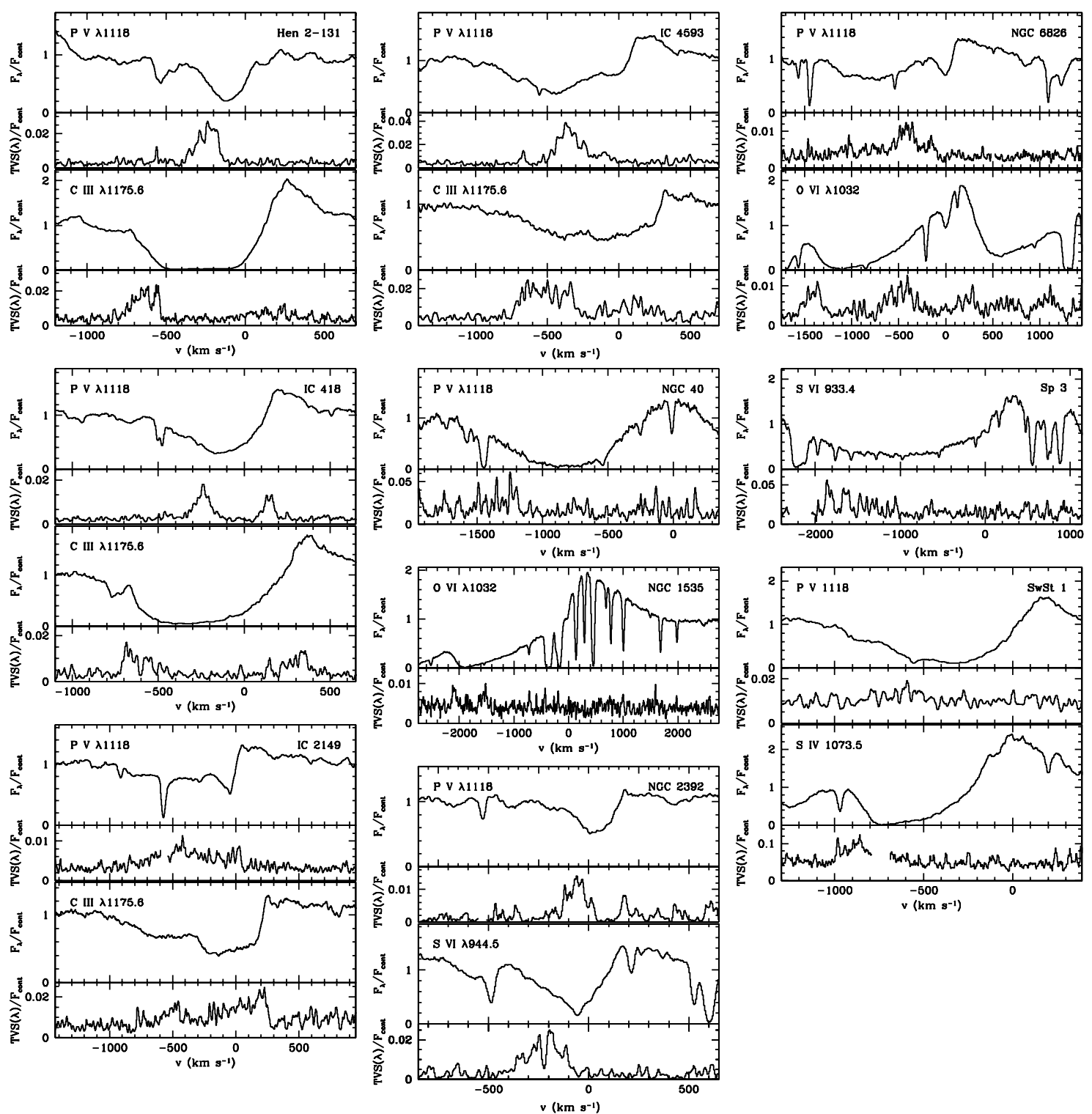

Fig. 5. Selected P Cygni profiles of the CSPNe showing FUSE variability. For each line, the top panel shows the averaged spectrum and the lower panel the temporal variation spectrum, TVS; both are normalized by the continuum level at the spectral range of the line. Note that we have omitted the TVS at specific wavelength ranges where the continuum level of the averaged spectrum falls to zero, either in the trough of some lines (e.g., the S VI $2933.4 \AA$ line of Sp 3) or at the location of narrow, deep absorption lines (e.g., the P V $\lambda 1118 \AA$ line of IC 2149).

\subsubsection{Hen $2-131$}

Figure 6 plots the profiles of the P V $\lambda 1118$, Si III $\lambda 1113$, and Si IV $\lambda 1122$ lines of Hen 2-131 (bottom-panels) normalized by their mean spectrum (top-panels). The normalized spectra taken on 2000-06-28, 2006-06-28, 2006-06-29, 2007-04-15, 2007-0615 , and 2007-06-16 illustrate the long-term variability of these lines, while the individual exposures obtained on 2007-06-15 and 2007-06-16 illustrate their short-term variability.

All the individual normalized spectra of the $\mathrm{PV}$ and Si IV lines show a broad DAC that is persistently observed in the trough of the line at a velocity up to $90 \%$ of $v_{\infty}$. The Si III line also shows a feature at this velocity, but it is narrower $(\mathrm{N})$ than in the two other lines. The first two spectra taken on 2007-06-16 $(\Delta t \sim 5 \mathrm{~h}$ ) show the absorption moving bluewards. The feature is also clearly seen in the spectra taken on 2007-06-15 UT00:22:39 and 2007-06-17 UT17:32:36, i.e., $\sim 40 \mathrm{~h}$ apart. If we assume this corresponds to the same DAC moving bluewards, the observed shift in velocity imply an acceleration $\sim 1 \times 10^{-4} \mathrm{~km} \mathrm{~s}^{-2}$ for a terminal wind velocity of $370 \mathrm{~km} \mathrm{~s}^{-1}$.

Although there are IUE high-dispersion SWP data available for Hen 2-131 (SWP07653, SWP10854, and SWP10855), their $\mathrm{S} / \mathrm{N}$ is inadequate to search for time variability of the P Cygni profiles of the C IV $\lambda \lambda 1548,1551$ lines. Otherwise, the FUSE variability detected in this study can be linked to the occurrence of variable optical lines reported by Méndez (1989), although these variations were attributed to binary motions rather than to wind variability. The binarity claim has not been confirmed yet.

\subsubsection{IC 418}

Figure 7 plots the profiles of the P V $\lambda 1118$, Si IV $\lambda 1122$, and C III $\lambda 1176$ lines of IC 418 (bottom-panels) normalized by their mean spectrum (top-panels). All the three normalized spectra were taken on 2001-12-02 with a cadence $\sim 1.6 \mathrm{~h}$. 
M. A. Guerrero and O. De Marco: FUSE survey of CSPNe
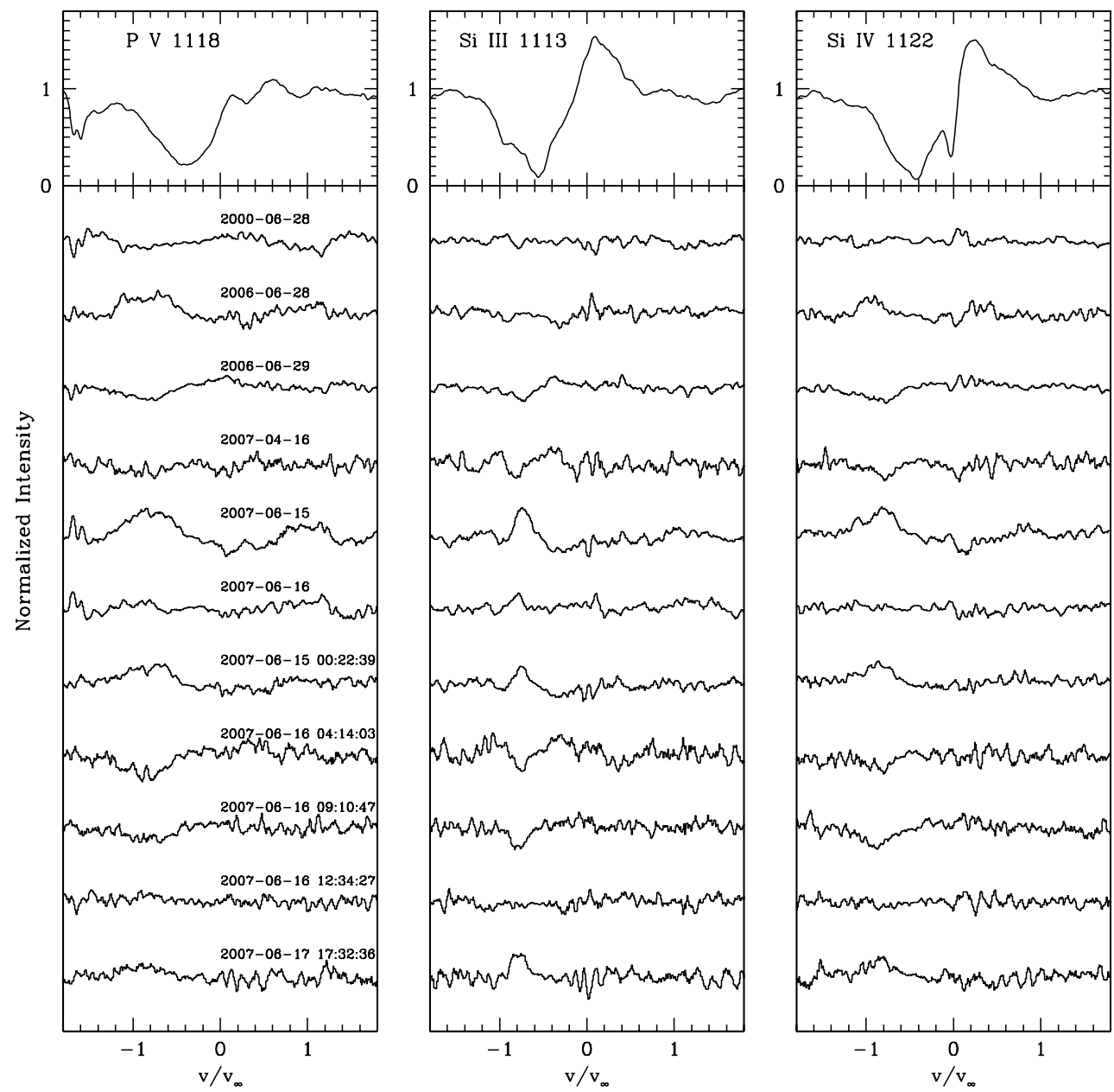

Fig. 6. Top-panels: averaged P V $\lambda 1118$ (left), Si III $\lambda 1113$ (center), and Si IV $\lambda 1122$ (right) P Cygni profiles of Hen 2-131. (Bottom-panels: normalized differences between individual spectra and the corresponding averaged spectrum shown in the top-panel. The epoch of each observation is indicated on the $\mathrm{P} \mathrm{V}$ spectra.
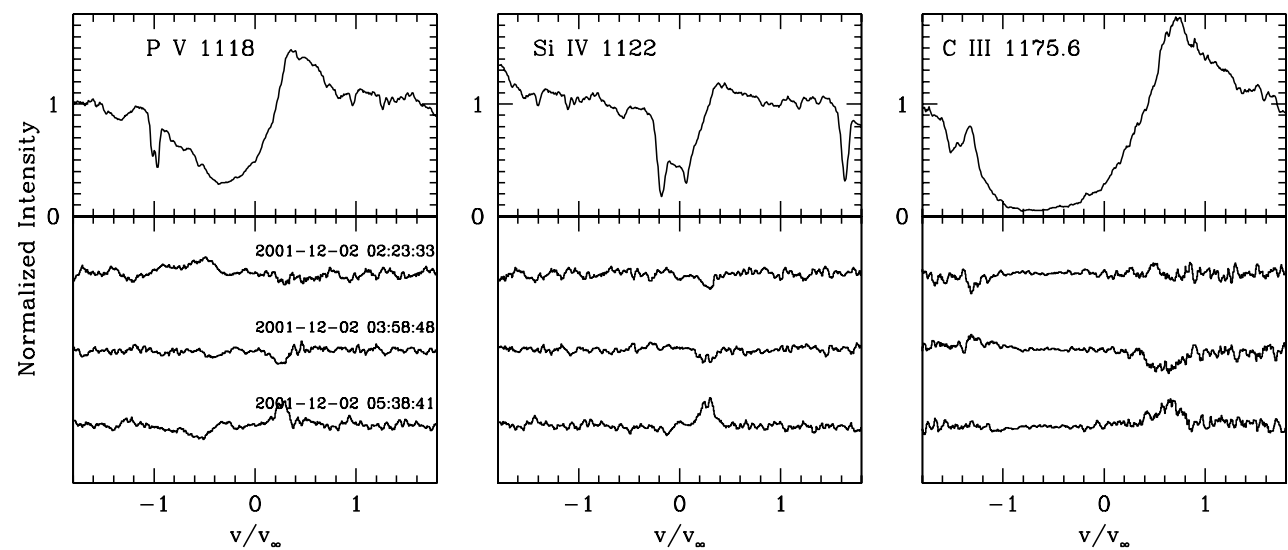

Fig. 7. Same as Fig. 6 for the P v $\lambda 1118$, Si IV $\lambda 1122$, and C III $\lambda 1176$ P Cygni profiles of IC 418.

As for Hen 2-131, the $\mathrm{P}$ Cygni profile of the $\mathrm{P} \mathrm{V}$ line of IC 418 shows a broad DAC that seems to move bluewards. For this feature, Prinja et al. (2012b) reports a velocity shift $\sim 100 \mathrm{~km} \mathrm{~s}^{-1}$. Contrary to Hen 2-131, the ripples in the $\mathrm{P} \mathrm{V}$ and C III P Cygni profiles of IC 418 extend to velocities bluer than $v_{\infty}(\mathrm{B})$. Moreover, these two lines, as well as the Si IV line, show variability of the emission region of the profile $(\mathrm{E})$.
The IUE observations of IC 418 detected P Cygni profiles of the Si IV $\lambda \lambda 1393,1401, \mathrm{C}$ IV $\lambda \lambda 1548,1551$, and N IV $\lambda 1719$ lines whose possible variability was discussed in detail and rejected by Patriarchi \& Perinotto (1995). We have examined the P Cygni profile of the C IV lines based on IUE SWP data obtained on December 8, 1989. This line shows some evidence of variability in the emission region (E), as well as a broad ripple at 
velocities above $v_{\infty}(\mathrm{B})$, although the quality of the IUE data is not sufficient to claim a firm detection of variability. We note that Méndez (1989) detected stellar emission and absorption lines moving in anti-phase that can be attributed to binary motion or to wind variability.

\subsubsection{IC 2149}

Figure 8 plots profiles of the P V $\lambda 1118$ line of IC 2149 (bottom-panels) normalized by its mean spectrum (top-panels). The normalized spectra taken on 1999-11-26, 1999-12-02, and 2000-01-14 show long-term variability, while the individual exposures obtained on $1999-12-02$ with a cadence $\sim 1.9 \mathrm{~h}$ illustrate the short-term variability.

The P Cygni profile of the P V line of IC 2149 show large variations within time scales as short as the spectral cadence. There is a notable broad DAC that seems to be traveling bluewards for which Prinja et al. (2012b) reports an acceleration $\sim 0.01 \mathrm{~km} \mathrm{~s}^{-2}$. Other spectra show also a broad feature at velocities up to $\sim 80 \%$ of $v_{\infty}$. There is some evidence of variability in the emission region of the profile $(\mathrm{E})$.

IUE SWP observations were obtained on August 1980, April 1982, and August 1983 and examined by Patriarchi \& Perinotto (1995). P Cygni profiles of the N V, Si IV, and $\mathrm{C}$ IV lines are detected, but no variability could be claimed from those data.

\subsubsection{IC 4593}

Figure 9 plots the profiles of the P V $\lambda 1118$, O VI $\lambda 1032$, and $\mathrm{C}$ III $\lambda 1176$ lines of IC 4593 (bottom-panels) normalized by their mean spectrum (top-panels). The two top profiles correspond to the mean spectra obtained on 2001-08-03 and 2004-04-16, respectively, whereas the following spectra are individual exposures obtained during these dates.

The P Cygni profiles of the three lines display noticeable DACs that move bluewards. On 2004-04-16, the DAC in the P V profile moved by $\sim 20 \%$ of $v_{\infty}$ over a period of $1.5 \mathrm{~h}$, implying an acceleration $\sim 0.03 \mathrm{~km} \mathrm{~s}^{-2}$, comparable to the measurement of $0.042 \mathrm{~km} \mathrm{~s}^{-2}$ reported by Prinja et al. (2012b). On these same observations, the ripple in the $\mathrm{O}$ VI lines extends beyond $v_{\infty}$ (B). No obvious variations of the emission region of the profile is detected.

IC 4593 was one of the CSPNe claimed to be variable by IUE (Patriarchi \& Perinotto 1995). The IUE C IV, O IV, Si IV, N IV, and $\mathrm{N} v$ lines show a behavior similar to that of the FUSE lines described above, i.e., little or no change in the emission region of the profile and variable troughs at velocities near or above $v_{\infty}$. De Marco et al. (2007) detected variability in the emission component of the He II $\lambda 4686 \AA$ line, whereby the entire emission line shifted to the red and the P Cygni trough of the line was also found to be variable. This behavior remains unexplained.

\subsubsection{NGC 40}

Figure 10 plots profiles of the P V $\lambda 1118$ line of NGC 40 (bottom-panel) normalized by its mean spectrum (top-panel). The two top normalized profiles correspond to the mean spectra obtained on 2000-09-14 and 2000-12-16, respectively, whereas the following two are individual exposures obtained on 2000-09-14. The separation in time between these two spectra is $\sim 8 \mathrm{~h}$.

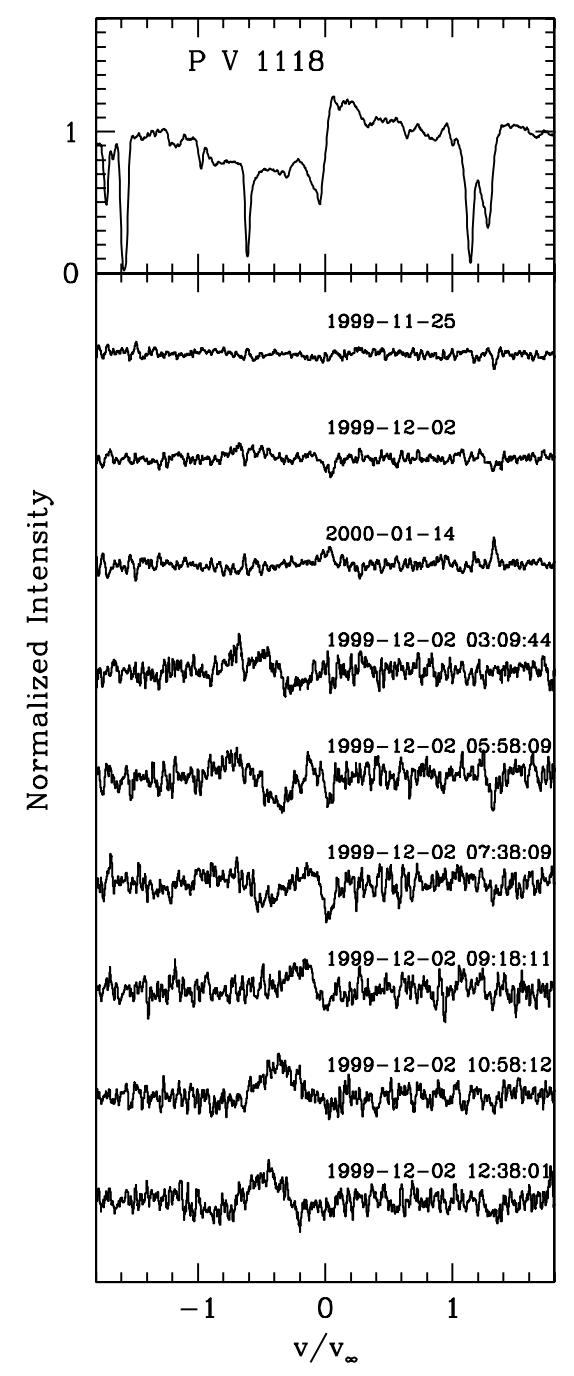

Fig. 8. Same as Fig. 6 for the P v $\lambda 1118$ P Cygni profile of IC 2149.

The P Cygni profile of the P V line of NGC 40 is rather flat, but it shows noticeable ripples above the terminal velocity $(\mathrm{R}, \mathrm{B})$ and on the emission region of the profile $(\mathrm{E})$.

NGC 40 was claimed to have non-variable P Cygni profiles by Patriarchi \& Perinotto (1995), based on IUE data acquired in January 1983 and August 1991. However, new IUE high resolution observations obtained on 1994-08-22 and 1994-11-06 revealed the variability of the P Cygni profiles of the $\mathrm{C}$ IV lines, while the $\mathrm{N} \mathrm{V}, \mathrm{O}$ V, and Si IV P Cygni profiles, also present in the IUE spectral range, were found to be constant (Patriarchi \& Perinotto 1997). As is the case for the FUSE P V line, the P Cygni profile of the IUE C IV line exhibits ripples at and above $v_{\infty}(\mathrm{R}, \mathrm{B})$ and on the emission region of the profile $(\mathrm{E})$.

\subsubsection{NGC 1535}

Figure 11 plots profiles of the O VI $\lambda 1037$ line of NGC 1535 (bottom-panel) normalized by its mean spectrum (top-panel). The normalized profiles correspond to the mean spectra obtained on 2001-10-05 and 2003-01-01, respectively.

The P Cygni profile of the O VI line of NGC 1535 shows a narrow feature at a velocity $\sim 10 \%$ above its wind terminal velocity of $1825 \mathrm{~km} \mathrm{~s}^{-1}(\mathrm{~N}, \mathrm{~B})$. No noticeable changes are observed in the emission region of the profile. The variability detected by FUSE does not match completely the changes observed in 
M. A. Guerrero and O. De Marco: FUSE survey of CSPNe
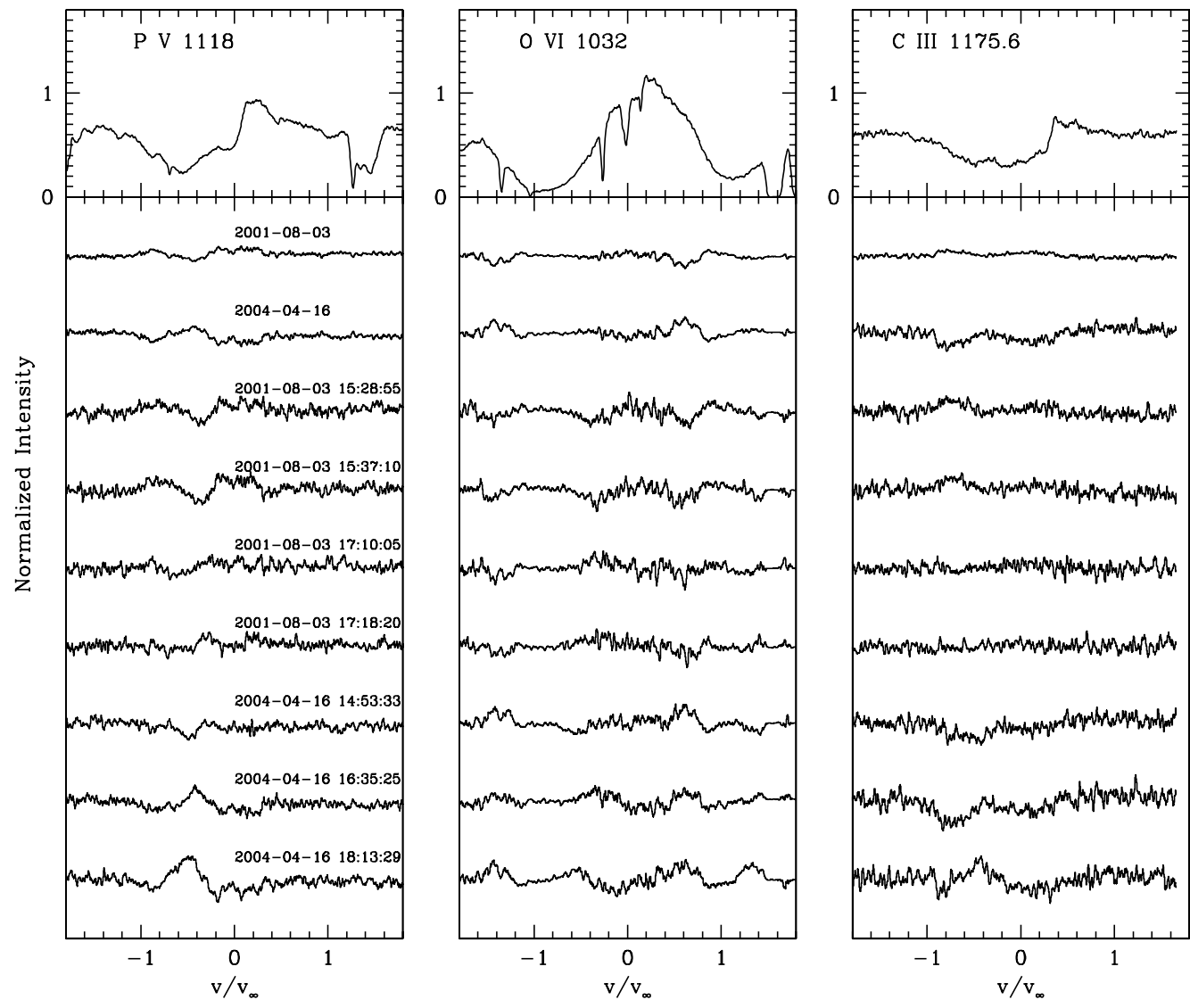

Fig. 9. Same as Fig. 6 for the P v $\lambda 1118$, O VI $\lambda 1032$, and C III $\lambda 1176$ P Cygni profile of IC 4593.

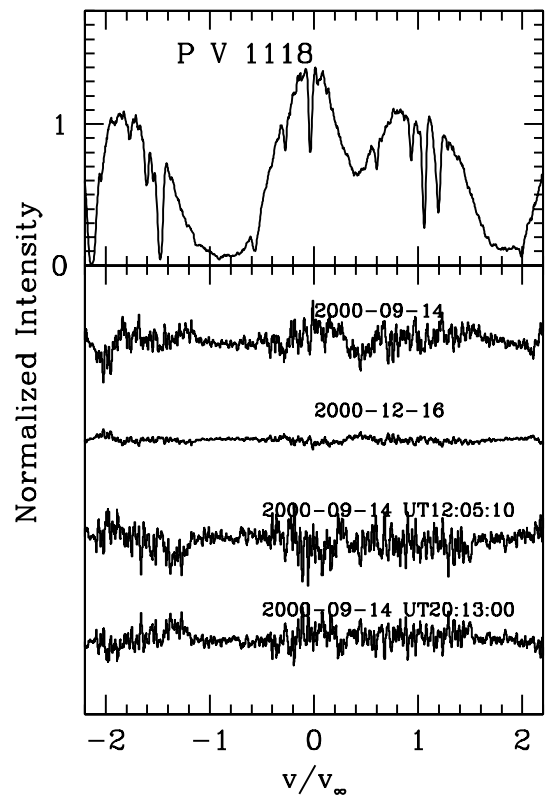

Fig. 10. Same as Fig. 6 for the P v $\lambda 1118$ P Cygni profile of NGC 40.

the N v P Cygni profiles by IUE between December 1980 and March 1981, as this line also shows variability of the emission region (Patriarchi \& Perinotto 1995).

\subsubsection{NGC 2392}

Figure 12 plots profiles of the P V $\lambda 1118$ and S VI $\lambda 944.5$ lines of NGC 2392 (bottom-panels) normalized by the mean spectrum

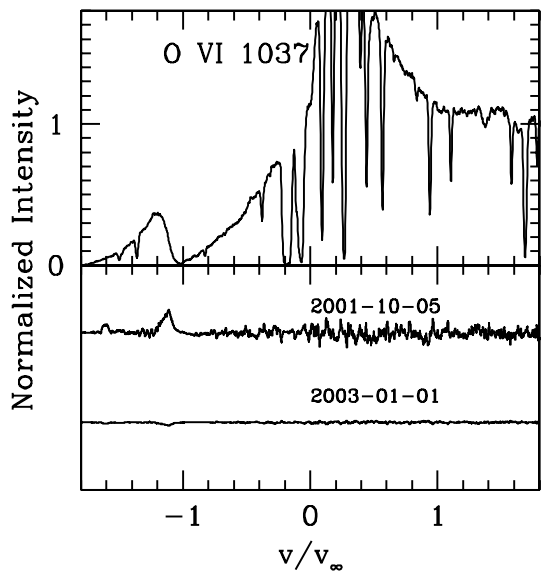

Fig. 11. Same as Fig. 6 for the O VI $\lambda 1037$ P Cygni profile of NGC 1535.

(top-panels). The normalized profiles correspond to individual exposures obtained on 2001-02-21 taken $\sim 1.85 \mathrm{~h}$ apart.

The P Cygni profiles of both the $\mathrm{P} \mathrm{V}$ and $\mathrm{S}$ VI lines of NGC 2392 show a broad ripple (R) that extends far bluewards of $v_{\infty}$ (B) due to its low value in this $\operatorname{CSPN}\left(v_{\infty}=65 \mathrm{~km} \mathrm{~s}^{-1}\right)$. We note that the ripple of these two lines are not completely in phase, with the peak of the $\mathrm{P} V$ line in the profile taken at UT16:27:25 at $\sim 3 \times v_{\infty}$, while that of the $\mathrm{S}$ VI line is at $\sim 7 \times v_{\infty}$.

The variability of the P Cygni profiles of NGC 2392 has already been reported by Patriarchi \& Perinotto (1995) based on IUE high-dispersion observations obtained on May 1983, and October and November 1990. The variability of the IUE C IV and $\mathrm{N} \mathrm{V}$ lines, however, affects the emission region of the 

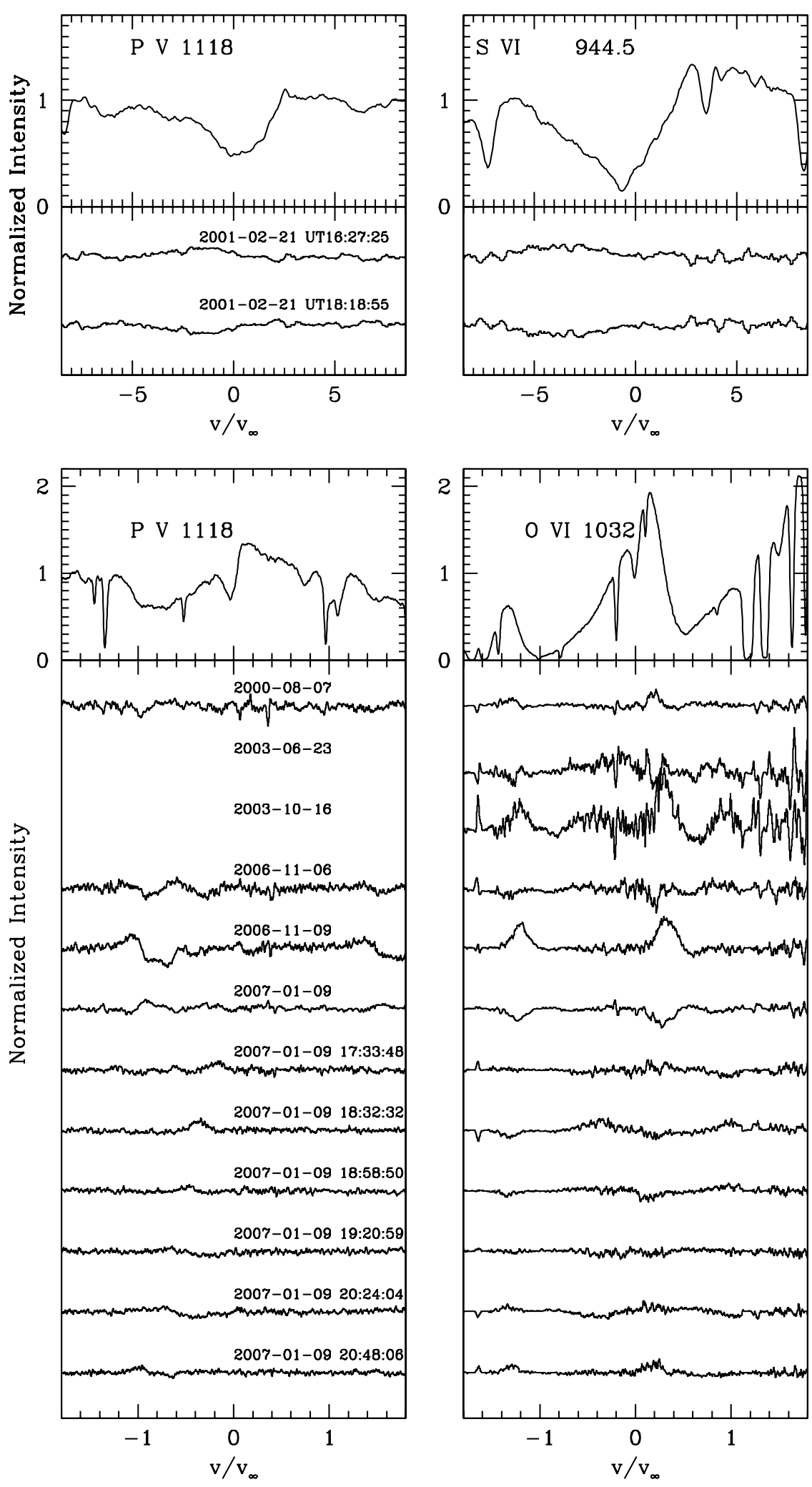

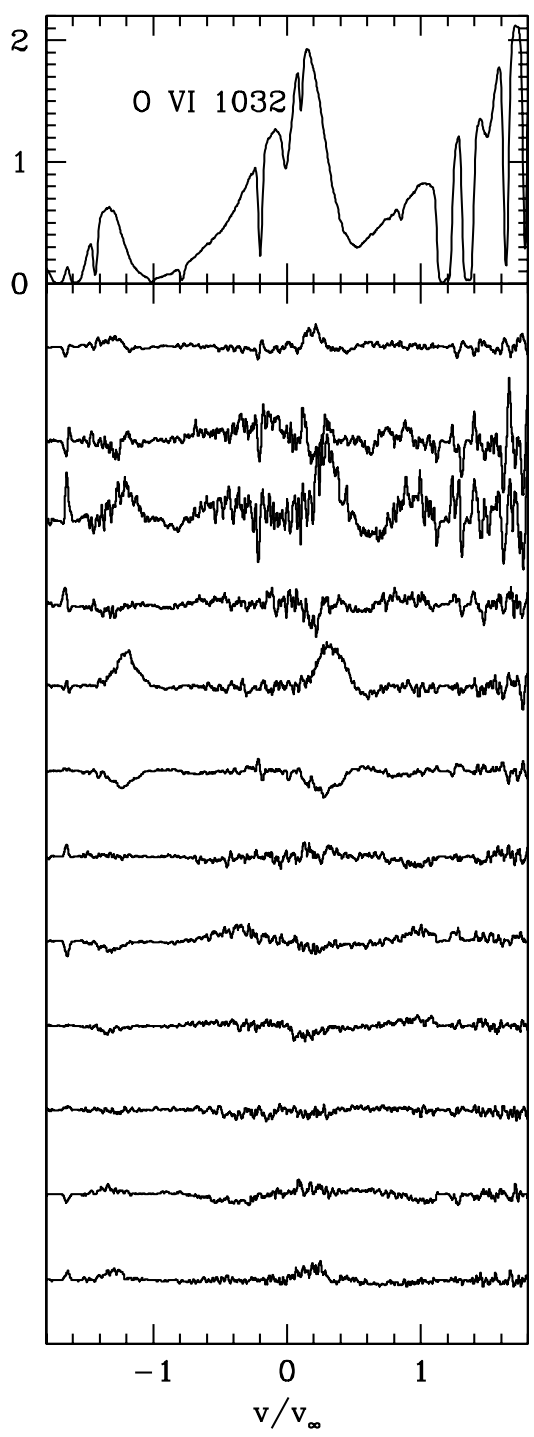

Fig. 12. Same as Fig. 6 for the P v $\lambda 1118$ and S VI 1944.5 P Cygni profiles of NGC 2392.
Fig. 13. Same as Fig. 6 for the P v $\lambda 1118$ and O VI $\lambda 1032$ P Cygni profiles of NGC 6826. profiles (E), while the FUSE variability is better described as a broad ripple $(\mathrm{R})$ at velocities well above $v_{\infty}(\mathrm{B})$, but with no variability in the emission region of the profile.

\subsubsection{NGC 6826}

Figure 13 plots profiles of the P V $\lambda 1118$ and O VI $\lambda 1032$ lines of NGC 6826 (bottom-panels) normalized by the mean spectrum (top-panels). The normalized profiles correspond to mean spectra obtained on 2000-08-07, 2003-06-23, 2003-1016, 2006-11-06, 2006-11-09, and 2007-11-09, but we note that no suitable observations of the $\mathrm{P} \mathrm{V}$ line are available for the observations obtained in 2003 (2003-06-23 and 2003-10-16). The spectra obtained on the 2007-11-09 has further been split into 6 individual exposures acquired with a cadence between 0.5 and $1.0 \mathrm{~h}$. 

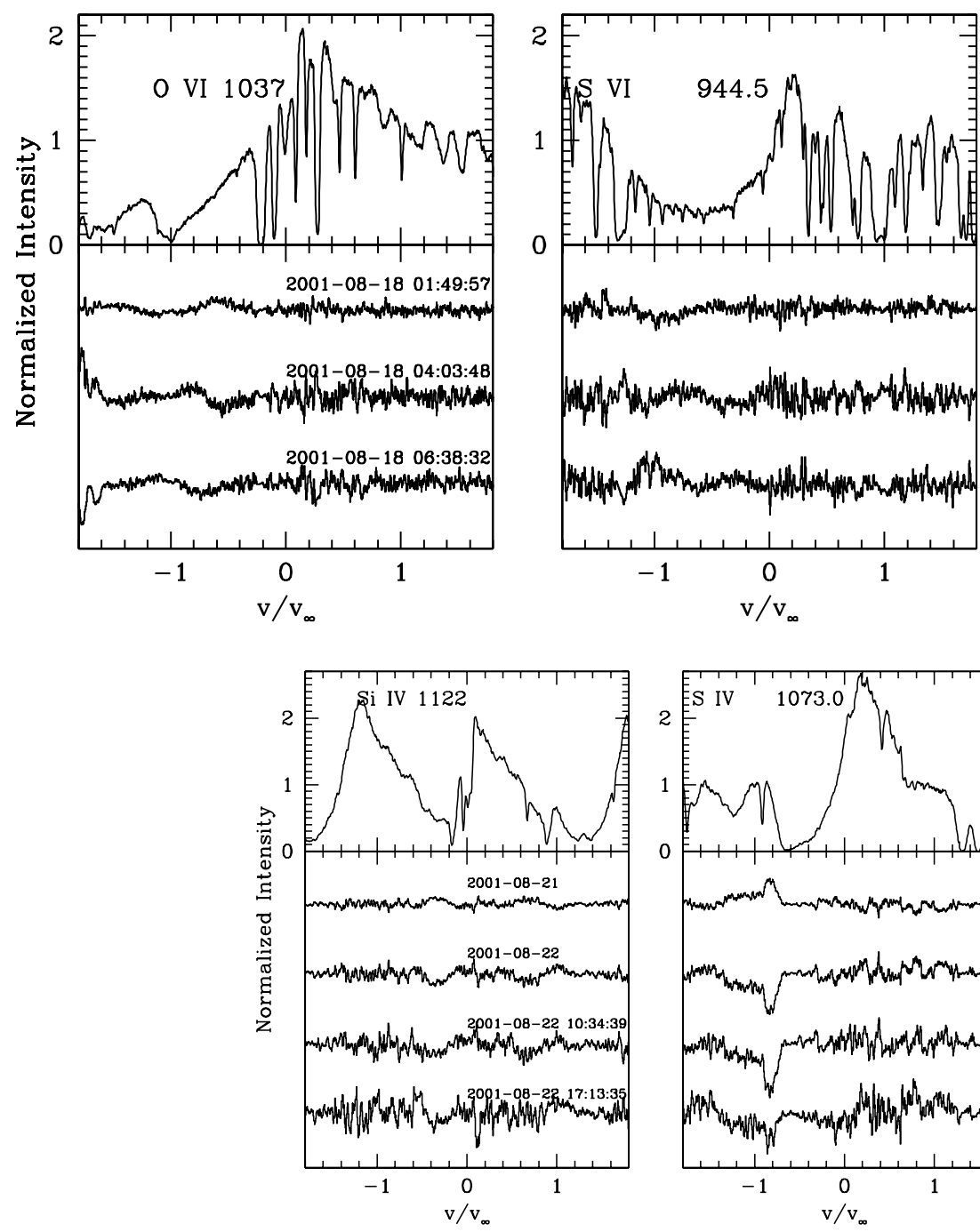

Fig. 14. Same as Fig. 6 for the O VI $\lambda 1032$ and S VI $\lambda 944.5$ P Cygni profiles of $\mathrm{Sp} 3$.

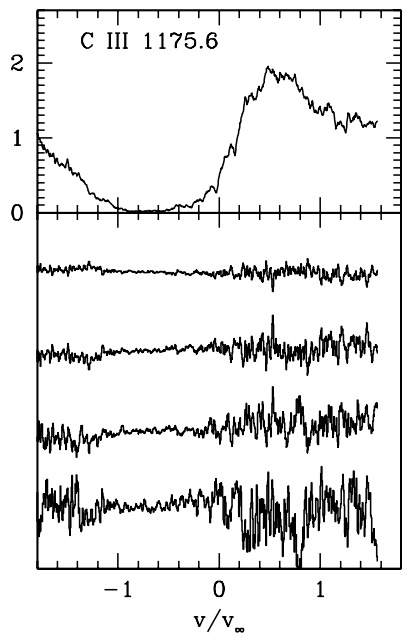

Fig. 15. Same as Fig. 6 for the Si IV $\lambda 1122$, S IV $\lambda 1073$, and C III $\lambda 1176$ P Cygni profiles of SwSt 1.

The P Cygni profile of the O VI line of NGC 6826 show persistent narrow features $(\mathrm{N})$ that are found at velocities above the terminal velocity (B). Broad DACs are also traveling across the profile, as seen in the $\mathrm{P}$ Cygni profile of the $\mathrm{P} \mathrm{V}$ line. In particular, the individual exposures acquired on 2007-11-09 show a feature that moves from a velocity $\sim 0.2 \times v_{\infty}$ (i.e., $\sim 200 \mathrm{~km} \mathrm{~s}^{-1}$ ) up to $\sim 1.0 \times v_{\infty}$ (i.e., $1100 \mathrm{~km} \mathrm{~s}^{-1}$ ), thus implying a velocity change of $\sim 900 \mathrm{~km} \mathrm{~s}^{-1}$ in a period of $5.25 \mathrm{~h}$, or an averaged acceleration $\sim 0.05 \mathrm{~km} \mathrm{~s}^{-2}$ in perfect agreement with the measurement by Prinja et al. (2012b).

The variability of the P Cygni profiles of the IUE C IV, N IV, and N V lines of NGC 6826 was reported by Patriarchi \& Perinotto $(1995,1997)$ based on high-resolution observations acquired in July and August 1982, February 1986, August 1994, and September 1995. All the variations observed in the profiles of the $\mathrm{C}$ IV and $\mathrm{N} \mathrm{V}$ lines affect the trough at velocities close to or above $v_{\infty}$, while the changes observed in the unsaturated N IV line would be better described as a broad ripple (R). Additional photometric and spectroscopic variations have been interpreted in terms of wind variability (Handler et al. 2013).

\subsection{9. $\mathrm{Sp} 3$}

Figure 14 plots profiles of the O VI $\lambda 1037$ and S VI $\lambda 944.5$ lines of Sp 3 (bottom-panels) normalized by the mean spectrum (top-panels). The normalized profiles correspond to individual exposures obtained on 2001-08-18, taken with a cadence $\sim 2.4 \mathrm{~h}$.

The P Cygni profiles of both the O VI and S VI lines of Sp 3 show a broad ripple $(\mathrm{R})$ traveling bluewards through the profile trough up to velocities bluewards of $v_{\infty}$ (B). The peak of these ripples are not completely in phase, as the $\mathrm{O}$ VI ripple is $(0.1-0.2) \times v_{\infty}$ bluewards of the $\mathrm{S}$ VI ripple, but they move coherently, with a change of velocity $\sim 0.5 \times v_{\infty}$ (i.e., $\sim 800 \mathrm{~km} \mathrm{~s}^{-1}$ ) in a period $\sim 4.8 \mathrm{~h}$. This implies an averaged acceleration of $\sim 0.05 \mathrm{~km} \mathrm{~s}^{-2}$, very similar to what is found in NGC 6826 .

\subsubsection{SwSt 1}

Figure 15 plots profiles of the Si IV $\lambda 1122, S$ IV $\lambda 1073$, and C III $\lambda 1176$ lines of SwSt 1 (bottom-panels) normalized by the mean spectrum (top-panels). The two top normalized profiles correspond to the mean spectra acquired on 2001-08-21 and 2001-08-22, respectively. The latter spectrum has been expanded into two consecutive individual exposures obtained $\sim 6.6 \mathrm{~h}$ apart.

The P Cygni profiles of the three lines show very different types of variability. The Si IV line shows a ripple (R) overimposed on its trough, with some evidence that this ripple is traveling outwards in the wind from one exposure to the next. On the other hand, the $\mathrm{S}$ IV profile shows a narrow feature $(\mathrm{N})$ at 

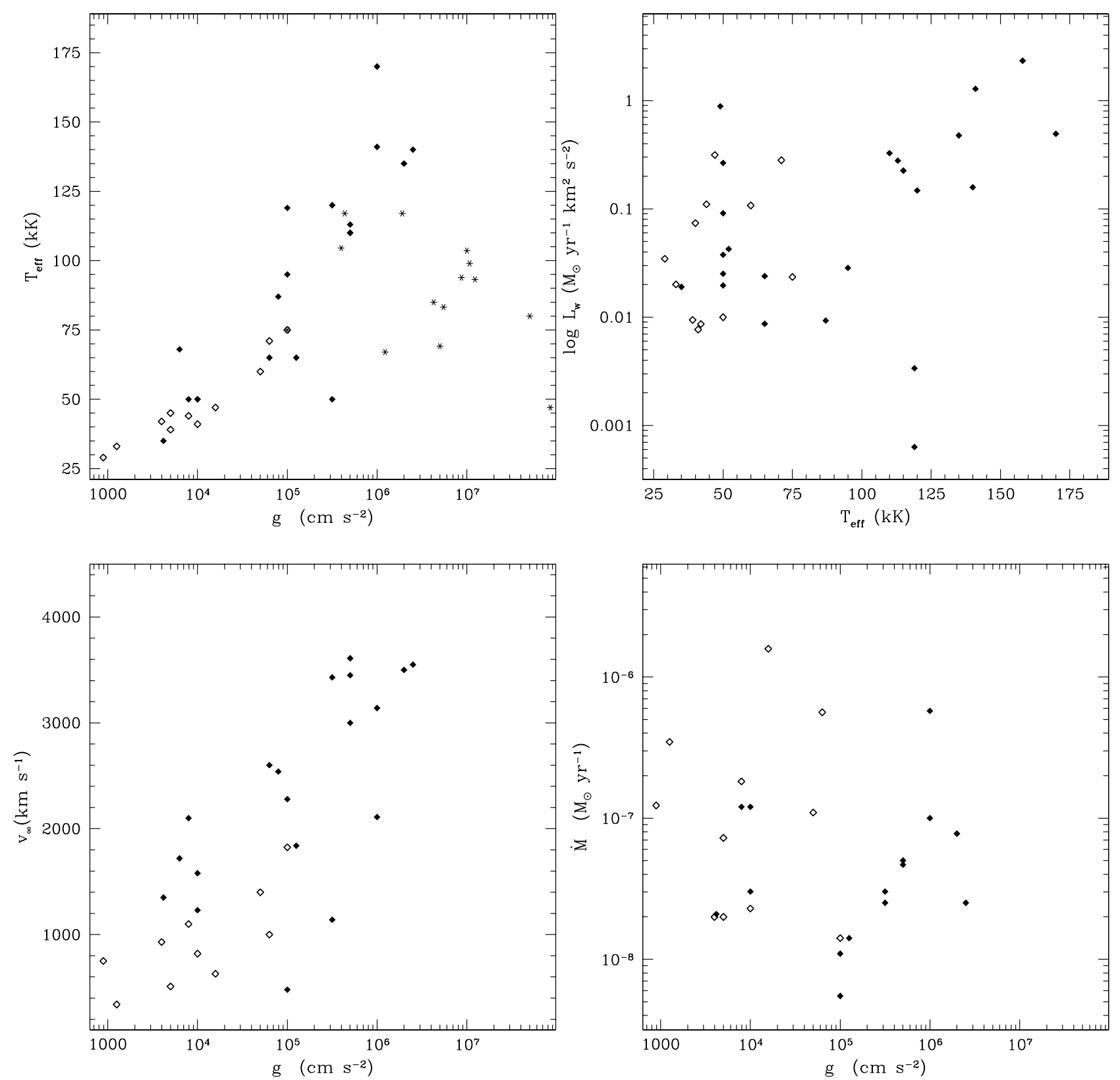

Fig. 16. Distributions of the CSPNe with P Cygni profile in FUSE spectra in the $T_{\text {eff }}-\log g$ (top-left), $L_{\text {wind }}-T_{\text {eff }}$ (top-right), $v_{\infty}-\log g$ (bottom-left), and $\log \dot{M}-\log g$ (bottom-right) diagrams. The CSPNe with variable P Cygni profiles are shown with open diamonds, while those without variable P Cygni profiles are shown with filled diamonds. In addition, the $T_{\mathrm{eff}}-\log g$ diagram (top-left) shows the location of the CSPNe without P Cygni profiles (stars).

$\sim 0.85 \times v_{\infty}$ with a "shoulder" extending bluewards of the terminal velocity (B). Finally, the changes of the C III line manifest as variations of the slope across the whole trough from one spectrum to another. As for many other sources in this study, there is no obvious evidence of change in the emission region of the profile.

\section{Discussion}

\subsection{Occurrence of wind variability}

In order to investigate whether the occurrence of wind variability correlates with stellar and wind parameters, we plot in Fig. 16 the location of the CSPNe with FUSE observations in different diagrams comparing stellar $\left(T_{\text {eff }}, \log g\right)$ and wind $(\dot{M}$, $\left.v_{\infty}, L_{\mathrm{w}}=\frac{1}{2} \dot{M} v_{\infty}^{2}\right)$ properties. An inspection of Fig. 16 reveals that the CSPNe with variable FUSE and/or IUE P Cygni profiles are to some extent clustered in all diagrams, as they tend to have low effective temperature $\left(T_{\text {eff }} \leq 70000 \mathrm{~K}\right)$, low gravity $(\log g \leq$ 5.3), and slow terminal velocities $\left(v_{\infty} \leq 2000 \mathrm{~km} \mathrm{~s}^{-1}\right)$.

It is, thus, tempting to conclude that the stellar winds of evolved CSPNe do not present variability. It must be kept in mind, however, that the P Cygni profiles of many of the evolved CSPNe with the highest $T_{\text {eff }}$ and $\log g$ are highly saturated (e.g., A 78 or IC 2448), as noted in Table 3 (quality code $N=2$ ). It would be fair to state that the search for wind variability among the evolved CSPNe does not reach the same sensitivity as for the less evolved CSPNe, thus potentially introducing a bias in the detection rate among CSPNe at different evolutionary stages. 
On the other hand, there are several CSPNe that, having very similar stellar and wind properties as those with variable P Cygni profiles, do not show any variability. Very notable examples are $\mathrm{Hb} 7$, Hen 2-99, IC 4776, NGC 5882, NGC 6058, NGC 6210, NGC 6891, PB 8, and Tc 1, as illustrated by Fig. 16 where they appear mixed among the CSPNe with variable P Cygni profiles in one or several of the diagrams. In most cases, the quality of the available data, as noted in Table 3 by $N=1$, may have hampered the detection of variability. The only CSPN that is definitely found to be non variable is NGC $6210(N=3$ in Table 3$)$ based on 4 exposures with a total integration time of $6.6 \mathrm{ks}$ obtained on August 2, 2000.

Among the sample of CSPNe with FUSE observations, there are several that are classified as [WR] stars. These stars are expected to have stronger winds and thus wind variability is more likely. $\mathrm{BD}+30^{\circ} 3639$ ([WC9]), NGC 40 ([WC8]), and SwSt 1 ([WC9pec]) show variable P Cygni profiles, while the more evolved [WC]-PG 1159 A 30, A 78, and NGC 2371-72, and Hen 2-99 ([WC9]), NGC 2867 ([WO1-2]), PB 6 ([WO1]), and PB 8 ([WN/WC]) do not. As we have already discussed above, the quality of the observations does not allow us to determine whether wind variability among [WR] CSPNe is a common phenomenon.

To summarize, it can be concluded that the detection of variable P Cygni profiles is more likely in less evolved CSPNe; the reason could be physical, namely that wind variability is associated to earlier evolutionary stages. Alternatively, variability could simply be more easily detected in denser winds, whereas the detection could be more difficult in fast, tenuous winds with saturated P Cygni profiles. While a stronger wind might appear to be obviously promoted by higher luminosities occurring in younger CSPNe, it is not clear that variability bears the same relation to luminosity given the lack of variability in some CSPNe in the same luminosity group. We also note that the entire range of mass-loss rates has both variable and non variable winds. The lack of detection of P Cygni profile variability among a number of CSPNe with similar stellar and wind properties to those found to be variable suggests that the wind variability may be an intermittent phenomenom, or that variability and mass-loss are not fully related. More sensitive and dedicated UV observations, such as those possible with the upcoming World Space Observatory-UV (WSO-UV), are required to shed light into these questions.

\subsection{Characteristics of time-variable features}

The variability observed in the P Cygni profiles of the CSPNe described in Sect. 5 displays significant variety from broad ripples to narrow features. Narrow features can be ascribed to DACs, similar to those detected in OB stars. The CSPNe that show variable narrow features are Hen 2-131, NGC 1535, NGC 6826, and SwSt 1. In all cases, the narrow features appear around the wind terminal velocity, with NGC 1535 and NGC 6826 having narrow features exceeding $v_{\infty}$ by $\sim 10 \%$ and $\sim 20 \%$, respectively.

The fragmentary time coverage of the observations used in this analysis makes it difficult to quantify the variations in velocity of the variable features seen in this sample of CSPNe. When velocity shifts are detected (e.g., broad ripples traveling bluewards, as in NGC 6826), typical accelerations are in the range $0.01-0.05 \mathrm{~km} \mathrm{~s}^{-2}$, with features traveling a few tenths of the corresponding terminal velocities in time intervals of a few hours. The time scales of the variable features of CSPNe to travel along the troughs of the P Cygni profiles seem shorter than those of
OB stars, which are typically in the range of a few days (e.g., Howarth et al. 1995; Massa et al. 1995; Fullerton et al. 1997). If we assume these velocity shifts are associated to the stellar rotation, as is the case for OB stars, and consequently we conclude that the rotation periods of CSPNe are of the order or greater than a few hours, then for typical radii $\left(0.2-0.5 R_{\odot}\right)$ and an assumed solid body rotation, the rotational velocities of our sample would be in the range $50-150 \mathrm{~km} \mathrm{~s}^{-1}$. These rotational velocities compare favorably with those measured in stars in transition from CSPN to WD (40-70 $\mathrm{km} \mathrm{s}^{-1}$, Rauch et al. 2004) and cover the range of rotational velocities recently derived for a sample of CSPNe also using FUSE data $\left(50-110 \mathrm{~km} \mathrm{~s}^{-1}\right.$, Prinja et al. 2012b,a).

\subsection{Correlation between hard X-rays and wind variability}

As for the strong stellar winds of OB stars (Lucy \& White 1980; Gayley \& Owocki 1995), the variability of the P Cygni profiles of a CSPN may be associated with shocks in its stellar wind. These shocks can also produce hard X-ray emission detected, e.g., in NGC 6543 (Guerrero et al. 2001). Therefore, a correlation between hard X-ray emission in CSPNe and wind variability may be expected. The search for such correlation is limited to the sample of 20 CSPNe with FUSE spectra and X-ray observations (this paper; Kastner et al. 2012). Among these CSPNe, three objects with variable FUSE P Cygni profiles are hard X-ray emitters (NGC 2392, NGC 6543, and NGC 6826), while three others (BD+30 3639 , IC 418, and NGC 40) are not (Kastner et al. 2000; Guerrero et al. 2001; Montez et al. 2005; Kastner et al. 2012, Guerrero et al., in prep.). Among the CSPNe without $\mathrm{P}$ Cygni profiles or non-variable $\mathrm{P}$ Cygni profiles, seven are found to lack hard X-ray emission (Hen 2-99, GJJC 1, NGC 1360, NGC 3132, NGC 3587, NGC 6720, and NGC 7662), whereas another seven (A 30, K 1-16, NGC 246, NGC 2371-2, NGC 7009, NGC 7094, and NGC 7293) are hard X-ray emitters (Guerrero et al. 2001, 2012; Kastner et al. 2012; Montez \& Kastner 2013), although their FUSE spectra are not always of sufficient quality to search for variability.

The correlation between hard X-ray CSPNe emission and wind variability is not conclusive. A further clue on a possible shock wind origin of the hard X-ray emission from CSPNe may be provided by the comparison between the IP of a line with P Cygni profile and its CSPN's $T_{\text {eff }}$ (Fig. 2). The CSPNe with the highest $T_{\text {eff }}$ show P Cygni profiles of species of the highest IP, such as Ne VII, while CSPNe of lower $T_{\text {eff }}$ display only P Cygni profiles of species of lower IP. This is the behavior expected for lines that have a photoionization origin. However, Fig. 2 also shows that the $\mathrm{O}$ VI line, while having a high IP, is found in stars with a wide range of effective temperatures (30000 K $<T_{\text {eff }}<170000 \mathrm{~K}$ ). Interestingly, five out of the six CSPNe with $T_{\mathrm{eff}}<45000 \mathrm{~K}$ that show this line also have variable P Cygni profiles ${ }^{2}$. This suggests that CSPNe with line variability tend to show lines with IP higher than expected for their $T_{\text {eff }}$, implying that an additional source of ionization may be present. Auger ionizations from X-ray photons produced in their stellar winds would naturally explain the additional ionization, as is the case in OB stars (Cassinelli \& Olson 1979).

We would also like to point out that two of the three sources with variable winds but no hard X-ray emission, namely $\mathrm{BD}+30^{\circ} 3639$ and $\mathrm{NGC} 40$, are stars of the [WC] type. If X-ray

2 The sixth CSPN being Tc 1, for which the available FUSE observations have too low a $\mathrm{S} / \mathrm{N}$ to detect variability in the $\mathrm{P}$ Cygni profiles of C III, O VI, and S IV. 
are self-absorbed in thick winds, then these two objects are likely to be undetected in X-ray. Note also that stars of the [WC] type do not display variable $\mathrm{O}$ VI lines, which may be associated to $\mathrm{X}$-ray-emitting hot gas.

\section{Summary}

We used the archive of FUSE far-UV spectroscopic observations of CSPNe to search for P Cygni profiles of high-excitation lines and to investigate their variability. For all spectral lines except those due to the $\mathrm{O}$ VI ion, there is a clear correlation between their presence, the IP of the originating ion, and the stellar effective temperature: the hottest CSPNe have only lines from ions with the highest IP, while the coolest CSPNe have lines from ions of low IP. This clearly implies a photoionization origin of the P Cygni profiles of these stars. Indeed, the detection of P Cygni profiles of S VI and Ne VII in CSPNe with effective temperatures above $40000 \mathrm{~K}$ and $100000 \mathrm{~K}$, respectively, shows that, contrary to OB stars, hot CSPNe can photoionize "super-ions". On the other hand, the formation mechanism for the O VI "super-ion" in CSPNe is unclear, as line profiles from this ion are found in hot CSPNe, as well as in CSPNe too cold to photoionize $\mathrm{O} \mathrm{V}$.

We built a FUSE atlas of P Cygni profiles of CSPNe. The $\mathrm{P}$ Cygni profiles of lines of the same CSPN may present very different shapes, making it difficult to estimate the terminal velocity of the wind. We have used the black velocity of saturated lines to estimate $v_{\infty}$, whereas for unsaturated P Cygni profiles we introduce the gray velocity, $v_{\text {gray }}$, the velocity of the blueward edge of the absorption region that has the lowest intensity, as a new indicator of $v_{\infty}$. A comparison with IUE measurements available in the literature reveals that terminal velocities derived from IUE data are usually overestimated as they rely on $v_{\text {edge }}$.

$\mathrm{P}$ Cygni profile variability in far-UV lines is reported for the first time for six CSPNe, namely Hen 2-131, NGC 40, NGC 1535, NGC 2392, Sp 3, and SwSt 1, joining the sample of six CSPNe previously reported in FUSE observations (Hen 2-138, IC 418, IC 2149, IC 4593, NGC 6543, and NGC 6826, Prinja et al. 2007, 2010, 2012b). Different types of variability are detected such as ripples and narrow features moving bluewards even at velocities above $v_{\infty}$, changes in the emission region of the profile and changes in the slope of the profile. Including those CSPNe reported to have variable P Cygni profiles in IUE observations, the number of CSPNe with these characteristics amounts to 13 .

Despite the lack of a tight correlation between the samples of hard X-ray CSPNe and those with variable stellar winds, we find evidence that shocks in the stellar winds of CSPNe produce additional ionization that can be ascribed to Auger ionizations from $\mathrm{X}$-ray photons. In particular, all CSPNe with low effective temperature, $\lesssim 45000 \mathrm{~K}$, that show variability in P Cygni profiles, also have $\mathrm{P}$ Cygni profiles from the $\mathrm{O}$ VI ion, even though the low effective temperature is not sufficient to photoionize $\mathrm{O} v$. This strongly suggests a twofold origin of the P Cygni profile in lines from O VI that depend on the temperature of the CSPN and its variability. Therefore, CSPNe with variable P Cygni profiles should be considered as prime candidates for future X-ray observations aimed at detecting hard X-ray emission.

Acknowledgements. M.A.G. acknowledges support by grants PR 2009-0342 (Programa Nacional de Movilidad de Recursos Humanos del Plan Nacional de I-D+i 2008-2011), AYA 2008-01934, and AYA 2011-29754-C03-02 of the Spanish Ministerio de Ciencia e Innovación (MICINN) cofunded by FEDER funds, and by a Distinguished Visitor grant of the Anglo-Australian
Observatory (AAO). He also acknowledges with gratitude the hospitality of the Macquarie University where this paper was prepared while on sabbatical leave. Dr. Quentin A. Parker is kindly acknowledged for his support, as well as Dr. David Frew for his useful comments and suggestions. We appreciate the helpful comments of an anonymous referee which help improve this manuscript. All of the data presented in this paper were obtained from the Mikulski Archive for Space Telescopes (MAST). MAST is located at the Space Telescope Science Institute (STScI) which is operated by the Association of Universities for Research in Astronomy, Inc., under NASA contract NAS5-26555. Support for MAST for non-HST data is provided by the NASA Office of Space Science via grant NNX09AF08G and by other grants and contracts.

\section{References}

Abbott, D. C. 1978, ApJ, 225, 893

Balick, B., \& Frank, A. 2002, ARA\&A, 40, 439

Cassinelli, J. P., \& Olson, G. L. 1979, ApJ, 554, L55

Cerruti-Sola, M., \& Perinotto, M. 1989, ApJ, 345, 339

Chu, Y.-H., Guerrero, M. A., Gruendl, R. A., Williams, R. M., \& Kaler, J. B. 2001, ApJ, 553, L69

De Marco, O. 2002, in Interacting Winds from Massive Stars, eds. A. F. J. Moffat, \& N. St-Louis, (San Francisco: ASP), ASP Conf. Proc., 260, 517

De Marco, O., \& Crowther, P. A. 1999, MNRAS, 306, 931

De Marco, O., Crowther, P. A., Barlow, M. J., Clayton, G. C., \& de Koter, A. 2001, MNRAS, 328, 527

De Marco, O., Bond, H. E., Harmer, D., \& Fleming, A. J. 2004, ApJ, 602, L93

De Marco, O., Wortel, S., Bond, H. E., \& Harmer, D. 2007, in Asymmetrical Planetary Nebulae IV, published online at http://www.iac.es/proyect/ apn4, article \#75

Dixon, W. V., Sahnow, D. J., Barrett, P. E., et al. 2007, PASP, 119, 527

Feibelman, W. A. 1997, ApJS, 112, 193

Feibelman, W. A. 1998, ApJS, 114, 263

Feibelman, W. A. 1999, ApJ, 525, 863

Fullerton, A. W., Gies, D. R., \& Bolton, C. T. 1996, ApJS, 103, 475

Fullerton, A. W., Massa, D. L., Prinja, R. K., Owocki, S. P., \& Cranmer, S. R. 1997, A\&A, 327, 699

Gauba, G., Parthasarathy, M., Nakada, Y., \& Fujii, T. 2001, A\&A, 373, 572

Gayley, K. G., \& Owocki, S. P. 1995, ApJ, 446, 801

Gesicki, K., Zijlstra, A. A., Acker, A., et al. 2006, A\&A, 451, 925

Guerrero, M. A., Chu, Y.-H., Gruendl, R. A., Williams, R. M., \& Kaler, J. B. 2001, ApJ, 553, L55

Guerrero, M. A., Ramos-Larios, G., \& Massa, D. 2010, PASA, 27, 210

Guerrero, M. A., Ruiz, N., Hamann, W.-R., et al. 2012, ApJ, 755, 129

Hajduk, M., Zijlstra, A. A., \& Gesicki, K. 2010, MNRAS, 406, 626

Handler, G., Prinja, R. K., Urbaneja, M. A., et al. 2013, MNRAS, 430, 2923

Harrington, J. P., \& Paltoglou, G. 1993, ApJ, 411, L103

Herald, J. E., \& Bianchi, L. 2002, ApJ, 580, 434

Herald, J. E., \& Bianchi, L. 2004, ApJ, 609, 378

Herald, J. E., \& Bianchi, L. 2011, MNRAS, 417, 2440

Herald, J. E., Bianchi, L., \& Hillier, D. J. 2005, ApJ, 627, 424

Hoare, M. G., Barstow, M. A., Werner, K., \& Fleming, T. A. 1995, MNRAS, 273,812

Howarth, I. D., Bolton, C. T., Crowe, R. A., et al. 1993, ApJ, 417, 338

Howarth, I. D., Prinja, R. K., \& Massa, D. 1995, ApJ, 452, L65

Iping, R. C., Sonneborn, G., McCandliss, S. R., \& Chu, Y.-H. 2006, in Planetary Nebulae in our Galaxy and Beyond, eds. M. J. Barlow, \& R. H. Méndez, (Cambridge: Cambridge University Press), Proc. IAU Symp. 234, 429

Kastner, J. H., Soker, N., Vrtilek, S. D., \& Dgani, R. 2000, ApJ, 545, L57

Kastner, J. H., Montez, R., Jr., Balick, B., et al. 2012, AJ, 144, 58

Koesterke, L. 2001, Ap\&SS, 275, 41

Koesterke, L., \& Werner, K. 1998, ApJ, 500, L55

Koesterke, L., Dreizler, S., \& Rauch, T. 1998, A\&A, 330, 1041

Kwok, S., Purton, C. R., \& Fitzgerald, P. M. 1978, ApJ, 219, L125

Lau, H. H. B., De Marco, O., \& Liu, X.-W. 2011, MNRAS, 410, 1870

Leuenhagen, U., Hamann, W.-R., \& Jeffery, C. S. 1996, A\&A, 312, 167

Lucy, L. B., \& White, R. L. 1980, ApJ, 241, 300

Marcolino, W. L. F., Hillier, D. J., de Araujo, F. X., \& Pereira, C. B. 2007a, ApJ, 654,1068

Marcolino, W. L. F., de Araújo, F. X., Junior, H. B. M., \& Duarte, E. S. 2007b, AJ, 134, 1380

Massa, D., Fullerton, A. W., Nichols, J. S., et al. 1995, ApJ, 452, L53

Massa, D., Fullerton, A. W., Sonneborn, G., \& Hutchings, J. B. 2003, ApJ, 586, 996

McCarthy, J. K., Mould, J. R., Méndez, R. H., et al. 1990, ApJ, 351, 230

Méndez, R. 1989, in Planetary Nebulae, IAU Symp., 131, 261

Méndez, R. H., Herrero, A., \& Manchado, A. 1990, A\&A, 229, 152

Miksa, S., Deetjen, J. L., Dreizler, S., et al. 2002, A\&A, 389, 953 
M. A. Guerrero and O. De Marco: FUSE survey of CSPNe

Modigliani, A., Patriarchi, P., \& Perinotto, M. 1993, ApJ, 415, 258

Montez, R., Jr., \& Kastner, J. H. 2013, ApJ, 766, 26

Montez, R., Kastner, J. H., De Marco, O., \& Soker, N. 2005, ApJ, 635, 381

Montez, R., Jr., De Marco, O., Kastner, J. H., \& Chu, Y.-H. 2010, ApJ, 721, 1820

Motch, C., Werner, K., \& Pakull, M. W. 1993, A\&A, 268, 561

Moos, H. W., Cash, W. C., Cowie, L. L., et al. 2000, ApJ, 538, L1

Napiwotzki, R. 1999, A\&A, 350, 101

Napiwotzki, R., \& Schönberner, D. 1995, A\&A, 301, 545

Olson, G. L. 1981, ApJ, 245, 1054

Patriarchi, P., \& Perinotto, M. 1991, A\&AS, 91, 325

Patriarchi, P., \& Perinotto, M. 1995, A\&AS, 110, 353

Patriarchi, P., \& Perinotto, M. 1996, A\&A, 309, 895

Patriarchi, P., \& Perinotto, M. 1997, A\&AS, 126, 385

Pauldrach, A. W. A., Hoffmann, T. L., \& Méndez, R. H. 2004, A\&A, 419, 111

Peña, M., Ruiz, M. T., Rojo, P., Torres-Peimbert, S., \& Hamann, W.-R. 2008, ApJ, 680, L109

Perinotto, M., Schönberner, D., Steffen, M., \& Calonaci, C. 2004, A\&A, 414, 993

Prinja, R. K. 1990, A\&A, 232, 119

Prinja, R. K., Barlow, M. J., \& Howarth, I. D. 1990, ApJ, 361, 607
Prinja, R. K., Hodges, S. E., Massa, D. L., Fullerton, A. W., \& Burnley, A. W. 2007, MNRAS, 382, 299

Prinja, R. K., Hodges, S. E., Urbaneja, M. A., \& Massa, D. L. 2010, MNRAS, 402, 641

Prinja, R. K., Massa, D. L., \& Cantiello, M. 2012a, ApJ, 759, L28

Prinja, R. K., Massa, D. L., Urbaneja, M. A., \& Kudritzki, R.-P. 2012b, MNRAS, 422, 314

Rauch, T., Heber, U., \& Werner, K. 2002, A\&A, 381, 1007

Rauch, T., Köper, S., Dreizler, S., et al. 2004, in Stellar Rotation, eds. A. Meader, \& P. Eenens (San Francisco: ASP) Proc. IAU Symp., 215, 573

Sahai, R., \& Trauger, J. T. 1998, AJ, 116, 1357

Sahnow, D. J., Moos, H. W., Ake, T. B., et al. 2000, ApJ, 538, L7

Sánchez-Contreras, C., Cortijo-Ferrero, C., Miranda, L. F., Castro-Carrizo, A., \& Bujarrabal, V. 2010, ApJ, 715, 143

St.-Louis, N., Willis, A. J., \& Stevens, I. R. 1993, ApJ, 415, 298

Steffen, M., Schönberner, D., \& Warmuth, A. 2008, A\&A, 489, 173

Todt, H., Peña, M., Hamann, W.-R., \& Gräfener, G. 2010, A\&A, 515, A83

Villaver, E., Manchado, A., \& García-Segura, G. 2002, ApJ, 581, 1204

Weidmann, W. A., \& Gamen, R. 2011, A\&A, 526, A6

Werner, K., Rauch, T., Reiff, E., Kruk, J. W., \& Napiwotzki, R. 2004, A\&A, 427, 685 